\title{
Effects of two inert scalar doublets on Higgs boson interactions and the electroweak phase transition
}

\author{
Amine Ahriche, ${ }^{1,2, *}$ Gaber Faisel,,${ }^{3,4 \dagger}$ Shu-Yu Ho, ${ }^{5, \$}$ Salah Nasri, ${ }^{6,8}$ and Jusak Tandean ${ }^{3, \|}$ \\ ${ }^{1}$ Department of Physics, University of Jijel, PB 98 Ouled Aissa, DZ-18000 Jijel, Algeria \\ ${ }^{2}$ The Abdus Salam International Centre for Theoretical Physics, Strada Costiera 11, I-34014 Trieste, Italy \\ ${ }^{3}$ Department of Physics and Center for Theoretical Sciences, National Taiwan University, \\ Taipei 106, Taiwan \\ ${ }^{4}$ Egyptian Center for Theoretical Physics, Modern University for Information and Technology, \\ Cairo 11212, Egypt \\ ${ }^{5}$ Department of Physics, California Institute of Technology, Pasadena, California 91125, USA \\ ${ }^{6}$ Physics Department, UAE University, POB 17551 Al Ain, United Arab Emirates
}

(Received 24 June 2015; published 31 August 2015)

\begin{abstract}
We study some implications of the presence of two new scalar weak doublets beyond the standard model which have zero vacuum expectation values and are charged under an extra Abelian gauge symmetry. The additional gauge sector does not couple directly to standard-model particles. We investigate specifically the effects of the scalars on oblique electroweak parameters and on the interactions of the $125 \mathrm{GeV}$ Higgs boson, especially its decay modes $h \rightarrow \gamma \gamma, \gamma Z$ and trilinear self-coupling, all of which will be probed with improved precision in future Higgs measurements. Moreover, we explore how the new scalars may give rise to strongly first-order electroweak phase transition and also show its correlation with sizable modifications to the Higgs trilinear self-coupling.
\end{abstract}

DOI: $10.1103 /$ PhysRevD.92.035020

PACS numbers: 12.60.-i, 12.60.Fr, 14.80.Bn, 98.80.Cq

\section{INTRODUCTION}

The recent discovery [1,2] at the Large Hadron Collider (LHC) of a Higgs boson with mass around $125 \mathrm{GeV}$ and other properties consistent with the expectations of the standard model (SM) serves as yet another confirmation that it is a remarkably successful theory. Nevertheless, it is widely believed that new physics beyond it is still necessary at least to account for the compelling experimental evidence for neutrino mass and the astronomical indications of dark matter [3].

Among a great many possibilities beyond the SM are those with enlarged scalar sectors. Scenarios incorporating a second Higgs doublet are of course highly popular in the literature $[4,5]$. Of late models with three scalar weak doublets have also been gaining interest [6-14], as they can provide dark matter (DM) candidates [13] and/or an important ingredient for the mechanism that generates neutrino mass [14].

Here we consider this three-scalar-doublet possibility, particularly that in which two of the doublets possess zero vacuum expectation values (VEVs). The theory also involves a new Abelian gauge symmetry under which these two doublets are charged, while SM particles are not. As a consequence, the extra scalar particles do not couple

\footnotetext{
*aahriche@ictp.it

gfaisel@hep1.phys.ntu.edu.tw

*sho3@caltech.edu

\$snasri@uaeu.ac.ae

"jtandean@yahoo.com
}

directly to a pair of exclusively SM fermions. Because of the absence of their VEVs and couplings to SM fermion pairs, these scalars have been termed inert in the literature [8]. However, being members of weak doublets, these scalars have interactions with SM gauge bosons at tree level. In addition, the gauge boson associated with the new gauge group is taken to have vanishing kinetic mixing with the hypercharge gauge boson. Accordingly, the additional gauge sector can be regarded as dark.

With these choices, the scalar sector of the theory corresponds to one of the three-scalar-doublet models catalogued and studied in Ref. [8] in terms of all possible allowed symmetries. In the present paper, we entertain the scenario described above and explore some implications of the presence of the inert scalars. Specifically, we analyze constraints on them from collider measurements on the Higgs boson and from electroweak precision data. In addition, we look at the potential impact of the scalars on the Higgs trilinear self-coupling, anticipating future experiments that will probe it sufficiently well. To evaluate the coupling, we will employ the Higgs effective potential derived at the oneloop level. Moreover, we examine how the new particles, which we choose to have sub-TeV masses, may give rise to strongly first-order electroweak phase transition (EWPT), which is needed for electroweak baryogenesis to explain the baryon asymmetry of the Universe. As it has been pointed out in the context of other models that the strength of EWPT could be correlated with sizable modifications to the Higgs trilinear self-coupling [15-19], our results will indicate how this may be realized in the presence of the new doublets. 
Due to their tree-level interactions with SM gauge and Higgs bosons, the lightest of the inert scalars cannot serve as good candidates for DM, as they annihilate into SM particles too fast and hence cannot produce enough relic abundance. To account for DM, one needs to have a more complete theory, but we assume that the additional ingredients responsible for explaining DM have negligible or no impact on our scalar sector of interest, so that they do not affect the results of this paper. ${ }^{1}$

The plan of the paper is as follows. In the next section, we describe the scalar Lagrangian and address some theoretical constraints on its parameters, especially from the requirements on vacuum stability. Since the extra scalar doublets couple to the standard Higgs and gauge bosons and include electrically-charged members, they contribute at the one-loop level to the Higgs decays $h \rightarrow \gamma \gamma$ and $h \rightarrow$ $\gamma Z$ which have been under intense investigation at the LHC, the former channel having also been observed. We determine their rates in Sec. III, where we also start our numerical analysis by exploring the charged scalars' impact on these processes. In Sec. IV, we calculate the contributions of the new doublets to the oblique electroweak observables $S$ and $T$, on which experimental information is available. Sections V and VI contain our treatment of the new scalars' effects on the trilinear Higgs couplings and on the electroweak phase transition, respectively. After deriving the relevant formulas, we perform further numerical work in these sections. In Sec. VII, we discuss additional results and make our conclusions after combining different relevant constraints. A few appendices contain more discussions and formulas.

\section{SCALAR SECTOR}

\section{A. Lagrangian}

Compared to the SM with the Higgs doublet $\Phi$, the scalar sector is expanded with the addition of two weak doublets, $\eta_{1}$ and $\eta_{2}$. The theory also possesses an extra Abelian gauge symmetry, $\mathrm{U}(1)_{D}$, under which $\eta_{1,2}$ carry charges +1 and -1 , respectively, whereas SM particles are not charged. Accordingly, one can express the renormalizable Lagrangian for the interactions of the scalars with each other and with the standard $\mathrm{SU}(2)_{L} \times \mathrm{U}(1)_{Y}$ gauge bosons, $W_{1,2,3}$ and $B$, as well as the $\mathrm{U}(1)_{D}$ gauge boson $C$, as

$$
\mathcal{L}=\left(\mathcal{D}^{\mu} \Phi\right)^{\dagger} \mathcal{D}_{\mu} \Phi+\left(\mathcal{D}^{\mu} \eta_{1}\right)^{\dagger} \mathcal{D}_{\mu} \eta_{1}+\left(\mathcal{D}^{\mu} \eta_{2}\right)^{\dagger} \mathcal{D}_{\mu} \eta_{2}-\mathcal{V},
$$

where the covariant derivative $\mathcal{D}^{\mu}=\partial^{\mu}+(i g / 2) \tau_{j} W_{j}^{\mu}+$ $i g_{Y} \mathcal{Q}_{Y} B^{\mu}+i g_{D} \mathcal{Q}_{C} C^{\mu}$ also contains the gauge couplings $g, g_{Y}$, and $g_{D}$, Pauli matrices $\tau_{1,2,3}$, and $\mathrm{U}(1)_{Y, D}$ charge operators $\mathcal{Q}_{Y, C}$, while the scalar potential is

$$
\begin{aligned}
\mathcal{V}= & \mu_{1}^{2} \Phi^{\dagger} \Phi+\mu_{21}^{2} \eta_{1}^{\dagger} \eta_{1}+\mu_{22}^{2} \eta_{2}^{\dagger} \eta_{2}+\frac{1}{2} \lambda_{1}\left(\Phi^{\dagger} \Phi\right)^{2}+\frac{1}{2} \lambda_{21}\left(\eta_{1}^{\dagger} \eta_{1}\right)^{2}+\frac{1}{2} \lambda_{22}\left(\eta_{2}^{\dagger} \eta_{2}\right)^{2} \\
& +\lambda_{31} \Phi^{\dagger} \Phi \eta_{1}^{\dagger} \eta_{1}+\lambda_{32} \Phi^{\dagger} \Phi \eta_{2}^{\dagger} \eta_{2}+\lambda_{41} \Phi^{\dagger} \eta_{1} \eta_{1}^{\dagger} \Phi+\lambda_{42} \Phi^{\dagger} \eta_{2} \eta_{2}^{\dagger} \Phi \\
& +\frac{1}{2}\left[\lambda_{5} \Phi^{\dagger} \eta_{1} \Phi^{\dagger} \eta_{2}+\lambda_{5}^{*} \eta_{1}^{\dagger} \Phi \eta_{2}^{\dagger} \Phi\right]+\lambda_{6} \eta_{1}^{\dagger} \eta_{1} \eta_{2}^{\dagger} \eta_{2}+\lambda_{7} \eta_{1}^{\dagger} \eta_{2} \eta_{2}^{\dagger} \eta_{1} .
\end{aligned}
$$

Thus $\mathcal{Q}_{C} \Phi=0$ and $\mathcal{Q}_{C} \eta_{1}\left(\eta_{2}\right)=+\eta_{1}\left(-\eta_{2}\right)$. The parameters $\mu_{1,2 a}^{2}$ and $\lambda_{1,2 a, 3 a, 4 a, 6,7}$ with $a=1,2$ are necessarily real because of the hermiticity of $\mathcal{V}$, whereas $\lambda_{5}$ can be rendered real using the relative phase between $\Phi$ and $\eta_{1,2}$. Assuming that the $\mathrm{U}(1)_{D}$ symmetry stays intact, after electroweak symmetry breaking we can write

$$
\begin{aligned}
\Phi & =\left(\begin{array}{c}
0 \\
\frac{1}{\sqrt{2}}(v+h)
\end{array}\right), \quad \eta_{a}=\left(\begin{array}{c}
H_{a}^{+} \\
\eta_{a}^{0}
\end{array}\right), \\
\sqrt{2} \eta_{a}^{0} & =\operatorname{Re} \eta_{a}^{0}+i \operatorname{Im} \eta_{a}^{0},
\end{aligned}
$$

where $h$ represents the physical Higgs boson, $v \simeq 246 \mathrm{GeV}$ is the vacuum expectation value (VEV) of $\Phi$, and $H_{a}^{+}$and

\footnotetext{
${ }^{1}$ In a recently proposed scotogenic model [14], the inert scalars participate in the mechanism to generate light neutrino masses via one-loop interactions with new fermions which include good DM candidates. In such a context, our results would likely be modified.
}

$\eta_{a}^{0}$ denote, respectively, the electrically charged and neutral components of $\eta_{a}$, which has no VEV.

From the terms in $\mathcal{V}$ that are quadratic in the fields, it is straightforward to extract the mass eigenstates of the scalars. Thus the masses of $h$ and $H_{1,2}^{ \pm}$at tree level are given by

$$
\hat{m}_{h}^{2}=\mu_{1}^{2}+\frac{3}{2} \lambda_{1} v^{2}, \quad m_{H_{a}^{ \pm}}^{2}=\mu_{2 a}^{2}+\frac{1}{2} \lambda_{3 a} v^{2} .
$$

The $\lambda_{5}$ part in Eq. (2) causes mixing between the electrically neutral components $\eta_{1}^{0}$ and $\eta_{2}^{0 *}$, which are then related to the mass eigenstates $\chi_{1}$ and $\chi_{2}$ according to

$$
\begin{aligned}
\left(\begin{array}{c}
\eta_{1}^{0} \\
\eta_{2}^{0 *}
\end{array}\right) & =\left(\begin{array}{cc}
c_{\theta} & s_{\theta} \\
-s_{\theta} & c_{\theta}
\end{array}\right)\left(\begin{array}{l}
\chi_{1} \\
\chi_{2}
\end{array}\right), \\
c_{\theta} & =\cos \theta, \quad s_{\theta}=\sin \theta, \\
\tan (2 \theta) & =\frac{\lambda_{5} v^{2}}{2 m_{H_{2}^{ \pm}}^{2}-2 m_{H_{1}^{ \pm}}^{2}+\left(\lambda_{42}-\lambda_{41}\right) v^{2}},
\end{aligned}
$$


the resulting eigenmasses being given by

$$
\begin{aligned}
m_{\chi_{1,2}}^{2}= & \frac{1}{2}\left(m_{H_{1}^{ \pm}}^{2}+m_{H_{2}^{ \pm}}^{2}\right)+\frac{1}{4}\left(\lambda_{41}+\lambda_{42}\right) v^{2} \\
& \mp \frac{1}{2} \sqrt{\left[m_{H_{2}^{ \pm}}^{2}-m_{H_{1}^{ \pm}}^{2}+\frac{1}{2}\left(\lambda_{42}-\lambda_{41}\right) v^{2}\right]^{2}+\frac{1}{4} \lambda_{5}^{2} v^{4}} .
\end{aligned}
$$

Hence the $\mathrm{U}(1)_{D}$ charges of $\chi_{1,2}$ are the same as (opposite in sign to) that of $\eta_{1}\left(\eta_{2}\right)$ and $m_{\chi_{1}} \leq m_{\chi_{2}}$.

Alternatively, instead of $\chi_{a}$, one can choose to deal with their real and imaginary parts,

$$
\mathcal{S}_{a}=\sqrt{2} \operatorname{Re} \chi_{a}, \quad \mathcal{P}_{a}=\sqrt{2} \operatorname{Im} \chi_{a},
$$

which are $C P$-even and $C P$-odd states, respectively, and share mass, $m_{\mathcal{S}_{a}}=m_{\mathcal{P}_{a}}=m_{\chi_{a}}$. From Eq. (5), one then has in matrix form

$$
\left(\begin{array}{c}
\operatorname{Re} \eta_{1}^{0} \\
\operatorname{Re} \eta_{2}^{0} \\
\operatorname{Im} \eta_{1}^{0} \\
\operatorname{Im} \eta_{2}^{0}
\end{array}\right)=\left(\begin{array}{cccc}
c_{\theta} & s_{\theta} & 0 & 0 \\
-s_{\theta} & c_{\theta} & 0 & 0 \\
0 & 0 & c_{\theta} & s_{\theta} \\
0 & 0 & s_{\theta} & -c_{\theta}
\end{array}\right)\left(\begin{array}{c}
\mathcal{S}_{1} \\
\mathcal{S}_{2} \\
\mathcal{P}_{1} \\
\mathcal{P}_{2}
\end{array}\right),
$$

where the mixing matrix is orthogonal.

Later on, to simplify the analysis, we will concentrate on the scenario in which $\lambda_{5}$ is negligible compared to the other $\lambda$ 's in $\mathcal{V}$. In that case, as Eq. (5) indicates, the $\eta_{1}^{0}-\eta_{2}^{0 *}$ mixing is small, $\theta \ll 1$, provided that $\lambda_{5} v^{2} \ll 2 m_{H_{1}^{ \pm}}^{2}-2 m_{H_{2}^{ \pm}}^{2}+$ $\left(\lambda_{41}-\lambda_{42}\right) v^{2}$. Furthermore, one can see from Eq. (6) that at the same time $\chi_{1}$ and $\chi_{2}$ can be close in mass if $\frac{1}{2}\left|\lambda_{5}\right| v^{2} \ll\left|m_{H_{1}^{ \pm}}^{2}-m_{H_{2}^{ \pm}}^{2}+\frac{1}{2}\left(\lambda_{41}-\lambda_{42}\right) v^{2}\right| \ll m_{\chi_{1}}^{2}$.

\section{B. Theoretical constraints}

The parameters of the scalar potential are subject to a number of theoretical constraints. The stability of the vacuum implies that $\mathcal{V}$ must be bounded from below. As shown in Appendix A, with $\lambda_{5}$ being negligible, this entails that for $a=1,2$

$$
\begin{aligned}
& \lambda_{1}>0, \quad \lambda_{2 a}>0, \quad \lambda_{3 a}+\lambda_{4 a}^{0}+\sqrt{\lambda_{1} \lambda_{2 a}}>0, \quad \lambda_{6}+\lambda_{7}^{0}+\sqrt{\lambda_{21} \lambda_{22}}>0, \\
& \sqrt{\lambda_{1} \lambda_{21} \lambda_{22}}+\sqrt{\lambda_{1}}\left(\lambda_{6}+\lambda_{7}^{0}\right)+\sqrt{\lambda_{21}}\left(\lambda_{32}+\lambda_{42}^{0}\right)+\sqrt{\lambda_{22}}\left(\lambda_{31}+\lambda_{41}^{0}\right) \\
& \quad+\left[2\left(\sqrt{\lambda_{1} \lambda_{21}}+\lambda_{31}+\lambda_{41}^{0}\right)\left(\sqrt{\lambda_{1} \lambda_{22}}+\lambda_{32}+\lambda_{42}^{0}\right)\left(\sqrt{\lambda_{21} \lambda_{22}}+\lambda_{6}+\lambda_{7}^{0}\right)\right]^{1 / 2}>0,
\end{aligned}
$$

where $\lambda_{x}^{0} \equiv \operatorname{Min}\left(0, \lambda_{x}\right)$.

The $\mu^{2}$ and $\lambda$ parameters in $\mathcal{V}$ also need to have such values that its minimum with the VEV of $\Phi\left(\eta_{a}\right)$ being nonzero (zero) is global. This is already guaranteed [8] by the positivity of the mass eigenvalues in Eqs. (4) and (6).

In addition, the perturbativity of the theory implies that the magnitudes of the $\lambda$ parameters need to be capped. Thus, in numerical work our choices for their ranges, to be specified later on, will meet the general requirement $\left|\lambda_{x}\right|<8 \pi$, in analogy to that in the two-Higgs-doublet case [20].

\section{RESTRICTIONS FROM COLLIDER DATA}

The kinetic portion of the Lagrangian in Eq. (1) contains the interactions of the new scalars with the photon and weak bosons,

$$
\begin{aligned}
\mathcal{L} \supset & i H_{a}^{+} \stackrel{\leftrightarrow}{\partial}^{\mu} H_{a}^{-}\left(e A_{\mu}-g_{L} Z_{\mu}\right)+H_{a}^{+} H_{a}^{-}\left(e A-g_{L} Z\right)^{2}+\frac{i g}{2 c_{\mathrm{w}}}\left[c_{2 \theta}\left(\chi_{1}^{*} \stackrel{\leftrightarrow}{\partial}^{\mu} \chi_{1}-\chi_{2}^{*} \stackrel{\leftrightarrow}{\partial}^{\mu} \chi_{2}\right)+s_{2 \theta}\left(\chi_{1}^{*} \stackrel{\leftrightarrow}{\partial}^{\mu} \chi_{2}+\chi_{2}^{*} \stackrel{\leftrightarrow}{\partial}^{\mu} \chi_{1}\right)\right] Z_{\mu} \\
& +\frac{g^{2}}{4 c_{\mathrm{w}}^{2}} \chi_{a}^{*} \chi_{a} Z^{2}+\frac{i g}{\sqrt{2}}\left\{\left[c_{\theta}\left(H_{1}^{+} \stackrel{\leftrightarrow}{\partial}^{\mu} \chi_{1}^{*}+H_{2}^{+} \stackrel{\leftrightarrow}{\partial}^{\mu} \chi_{2}\right)+s_{\theta}\left(H_{1}^{+} \stackrel{\leftrightarrow}{\partial}^{\mu} \chi_{2}^{*}-H_{2}^{+} \stackrel{\leftrightarrow}{\partial}^{\mu} \chi_{1}\right)\right] W_{\mu}^{-}-\text {H.c. }\right\} \\
& +\frac{g^{2}}{2}\left(H_{a}^{+} H_{a}^{-}+\chi_{a}^{*} \chi_{a}\right) W^{+\mu} W_{\mu}^{-},
\end{aligned}
$$

where summation over $a=1,2$ is implicit,

$$
X \stackrel{\leftrightarrow}{\partial}^{\mu} Y=X \partial^{\mu} Y-Y \partial^{\mu} X, \quad g_{L}=\frac{g}{2 c_{\mathrm{w}}}\left(2 s_{\mathrm{w}}^{2}-1\right),
$$


$c_{\mathrm{w}}=\cos \theta_{\mathrm{w}}=\left(1-s_{\mathrm{w}}^{2}\right)^{1 / 2}$, with $\theta_{\mathrm{w}}$ being the usual Weinberg angle, $c_{2 \theta}=\cos (2 \theta)$, and $s_{2 \theta}=\sin (2 \theta)$. One can alternatively write Eq. (10) in terms of the real and imaginary components $\mathcal{S}_{a}$ and $\mathcal{P}_{a}$ of $\chi_{a}$, which becomes more lengthy and is relegated to Appendix B.

We now see that data from past colliders can lead to some constraints on the masses of the new scalars. Based on Eq. (10), we may infer from the experimental widths of the $W$ and $Z$ bosons and the absence so far of evidence for nonstandard particles in their decay modes that for $a, b=1,2$

$$
m_{H_{a}^{ \pm}}+m_{\chi_{b}}>m_{W}, \quad 2 m_{H_{a}^{ \pm}}>m_{Z}, \quad m_{\chi_{a}}+m_{\chi_{b}}>m_{Z} .
$$

The null results of direct searches for new particles at $e^{+} e^{-}$ colliders also imply lower limits on these masses, especially those of the charged scalars. ${ }^{2}$ For these reasons, in our numerical work we will generally consider the mass regions $m_{\chi_{a}} \geq 50 \mathrm{GeV}$ and $m_{H_{a}} \geq 100 \mathrm{GeV}$.

In addition to the requirements in the preceding paragraph and the vacuum stability conditions in Eq. (9), when selecting the inert scalars' parameters we take into account also the Higgs mass which will be estimated at the one-loop level in Sec. $\mathrm{V}$ and then limited to $m_{h}=(125.1 \pm 0.1) \mathrm{GeV}$, well within the ranges of the newest measurements [23,24]. More specifically, we will therefore make the parameter choices

$$
\begin{aligned}
0 & <\lambda_{2 a},\left|\lambda_{3 a}\right|,\left|\lambda_{4 a}\right|,\left|\lambda_{6}\right|,\left|\lambda_{7}\right|<3, \\
\left|\mu_{2 a}^{2}\right| & <(800 \mathrm{GeV})^{2}, \\
\left|\lambda_{5}\right| & <0.01 \mathrm{Min}\left(\lambda_{2 a},\left|\lambda_{3 a}\right|,\left|\lambda_{4 a}\right|,\left|\lambda_{6}\right|,\left|\lambda_{7}\right|\right) .
\end{aligned}
$$

The recently discovered Higgs boson may offer a window into physics beyond the SM. The presence of new particles can give rise to modifications to the standard decay modes of the Higgs and/or cause it to undergo exotic decays [25]. As data from the LHC will continue to accumulate with improving precision, they may uncover clues of new physics in the Higgs couplings or, otherwise, yield growing constraints on various models. Here we address some of the potential implications for our scenario of interest. Especially, the existing experimental information on the possible Higgs decay into invisible/nonstandard final states [26-30] and on the observed $h \rightarrow \gamma \gamma$ mode [24,31] can supply further restrictions on the inert scalars.

\footnotetext{
${ }^{2} \mathrm{~A}$ recent investigation [21] concerning the effects of the corresponding particles in the simplest scotogenic model [22] on the relevant processes measured at LEP II suggests that such charged scalars may face significant constraints if their masses are below $100 \mathrm{GeV}$.
}

The Higgs boson couples to a pair of them according to

$$
\begin{aligned}
\mathcal{L} \supset & \frac{2 h}{v}\left[\left(\mu_{21}^{2}-m_{H_{1}}^{2}\right) H_{1}^{+} H_{1}^{-}+\left(\mu_{22}^{2}-m_{H_{2}}^{2}\right) H_{2}^{+} H_{2}^{-}\right. \\
& +\left(c_{\theta}^{2} \mu_{21}^{2}+s_{\theta}^{2} \mu_{22}^{2}-m_{\chi_{1}}^{2}\right) \chi_{1}^{*} \chi_{1} \\
& +\left(c_{\theta}^{2} \mu_{22}^{2}+s_{\theta}^{2} \mu_{21}^{2}-m_{\chi_{2}}^{2}\right) \chi_{2}^{*} \chi_{2} \\
& \left.+c_{\theta} s_{\theta}\left(\mu_{21}^{2}-\mu_{22}^{2}\right)\left(\chi_{1}^{*} \chi_{2}+\chi_{2}^{*} \chi_{1}\right)\right]
\end{aligned}
$$

from the $\mathcal{V}$ part of Eq. (1). In view of the mass choices made above, it follows that the decay modes $h \rightarrow \chi_{a}^{*} \chi_{b}$, if kinematically allowed, contribute at tree level to the total width of the Higgs boson and are the leading channels into nonstandard final states in the model. Their rates have the form

$\Gamma\left(h \rightarrow \chi_{a}^{*} \chi_{b}\right)=\frac{\left|C_{\chi_{a}^{*} \chi_{b}}\right|^{2}}{4 \pi m_{h}^{3} v^{2}} \sqrt{\left(m_{h}^{2}-m_{\chi_{a}}^{2}-m_{\chi_{b}}^{2}\right)^{2}-4 m_{\chi_{a}}^{2} m_{\chi_{b}}^{2}}$,

where

$$
\begin{aligned}
& C_{\chi_{1}^{*} \chi_{1}}=c_{\theta}^{2} \mu_{21}^{2}+s_{\theta}^{2} \mu_{22}^{2}-m_{\chi_{1}}^{2}, \\
& C_{\chi_{2}^{*} \chi_{2}}=c_{\theta}^{2} \mu_{22}^{2}+s_{\theta}^{2} \mu_{21}^{2}-m_{\chi_{2}}^{2}, \\
& C_{\chi_{1}^{*} \chi_{2}}=C_{\chi_{2}^{*} \chi_{1}}=c_{\theta} s_{\theta}\left(\mu_{21}^{2}-\mu_{22}^{2}\right) .
\end{aligned}
$$

The combined branching ratio of these decays is

$$
\mathcal{B}\left(h \rightarrow \chi^{*} \chi^{\prime}\right)=\frac{\sum_{a, b} \Gamma\left(h \rightarrow \chi_{a}^{*} \chi_{b}\right)}{\Gamma_{h}^{\mathrm{SM}}+\sum_{a, b} \Gamma\left(h \rightarrow \chi_{a}^{*} \chi_{b}\right)},
$$

where $\Gamma_{h}^{\mathrm{SM}}$ is the SM Higgs total width and only channels satisfying $m_{\chi_{a}}+m_{\chi_{b}}<m_{h}$ contribute to the sums. Numerically, we adopt $\Gamma_{h}^{\mathrm{SM}}=4.08 \mathrm{MeV}$ [32] corresponding to $m_{h}=125.1 \mathrm{GeV}$. If these channels are open, we will require $\mathcal{B}\left(h \rightarrow \chi_{a}^{*} \chi_{b}\right)<0.19$, based on the latest analysis of the Higgs data [26-30].

The potential impact of the inert scalars can also be realized through loop diagrams. Of much interest are their contributions to the standard decay channels $h \rightarrow \gamma \gamma$ and $h \rightarrow \gamma Z$, which are already under investigation at the LHC. In the SM, they arise mainly from top-quark- and $W$-bosonloop diagrams. These modes receive additional contributions arising from the $H_{1,2}^{ \pm}$-loop diagrams drawn in Fig. 1, with vertices from Eqs. (10) and (14). ${ }^{3}$ Their decay rates are readily obtainable from those in the case of only one inert doublet [33]. Thus we get

\footnotetext{
${ }^{3}$ At the one-loop level, the charged (charged and neutral) inert scalars also induce $h \rightarrow \gamma C(h \rightarrow Z C, C C)$ involving the massless dark gauge boson $C$. These decay modes may be challenging to detect with $C$ being invisible, as their rates are expected to be roughly of similar order to those of the $\gamma \gamma$ and $\gamma Z$ channels.
} 

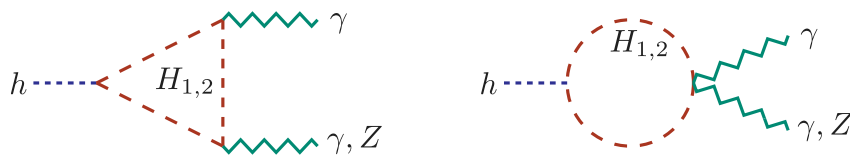

FIG. 1 (color online). Feynman diagrams for the contributions of the new charged scalars $H_{1,2}^{ \pm}$to the Higgs boson decays $h \rightarrow$ $\gamma \gamma$ and $h \rightarrow \gamma Z$. The triangle diagram with the gauge boson legs interchanged is not shown.

$$
\begin{aligned}
\Gamma(h \rightarrow \gamma \gamma)= & \frac{\alpha^{2} G_{F} m_{h}^{3}}{128 \sqrt{2} \pi^{3}} \mid \frac{4}{3} A_{1 / 2}^{\gamma \gamma}\left(\kappa_{t}\right)+A_{1}^{\gamma \gamma}\left(\kappa_{W}\right) \\
& +\left.\sum_{a=1}^{2} \frac{m_{H_{a}^{ \pm}}^{2}-\mu_{2 a}^{2}}{m_{H_{a}}^{2}} A_{0}^{\gamma \gamma}\left(\kappa_{H_{a}^{ \pm}}\right)\right|^{2}, \\
\Gamma(h \rightarrow \gamma Z)= & \frac{\alpha G_{F}^{2} m_{W}^{2}\left(m_{h}^{2}-m_{Z}^{2}\right)^{3}}{64 \pi^{4} m_{h}^{3}} \mid \frac{6-16 s_{\mathrm{w}}^{2}}{3 c_{\mathrm{w}}} A_{1 / 2}^{\gamma Z}\left(\kappa_{t}, \zeta_{t}\right) \\
& +c_{\mathrm{w}} A_{1}^{\gamma Z}\left(\kappa_{W}, \zeta_{W}\right) \\
& -\left.\frac{1-2 s_{\mathrm{w}}^{2}}{c_{\mathrm{w}}} \sum_{a=1}^{2} \frac{m_{H_{a}^{ \pm}}^{2}-\mu_{2 a}^{2}}{m_{H_{a}}^{2}} A_{0}^{\gamma Z}\left(\kappa_{H_{a}^{ \pm}}, \zeta_{H_{a}^{ \pm}}\right)\right|^{2},
\end{aligned}
$$

where $\alpha=g^{2} s_{\mathrm{w}}^{2} /(4 \pi)$ is the fine-structure constant, the expressions for the form factors $A_{0,1 / 2,1}^{\gamma \gamma, \gamma Z}$ are available from Ref. [34], the $A_{0}^{\gamma \gamma, \gamma Z}$ terms originate exclusively from the $H_{1,2}^{ \pm}$diagrams, $\kappa_{X}=4 m_{X}^{2} / m_{h}^{2}$, and $\zeta_{X}=4 m_{X}^{2} / m_{Z}^{2}$.

We can already test the new contributions to $h \rightarrow \gamma \gamma$, which has been observed at the LHC, unlike the $\gamma Z$ channel. For the $\gamma \gamma$ signal strengths, the ATLAS and CMS Collaborations measured $\sigma / \sigma_{\mathrm{SM}}=1.17 \pm 0.27$ [31] and $1.13 \pm 0.24$ [24], respectively. These numbers need to be respected by the ratio of $\Gamma(h \rightarrow \gamma \gamma)$ to its SM value,

$$
\mathcal{R}_{\gamma \gamma}=\frac{\Gamma(h \rightarrow \gamma \gamma)}{\Gamma(h \rightarrow \gamma \gamma)_{\mathrm{SM}}}
$$

Its $\gamma Z$ counterpart,

$$
\mathcal{R}_{\gamma Z}=\frac{\Gamma(h \rightarrow \gamma Z)}{\Gamma(h \rightarrow \gamma Z)_{\mathrm{SM}}},
$$

will be probed by future experiments.

To illustrate the effects of the inert scalars on $\mathcal{R}_{\gamma \gamma}$ and $\mathcal{R}_{\gamma Z}$, and possible (anti)correlation between them, we display in Fig. 2 the distribution of 5000 benchmark points on the $\left(\mathcal{R}_{\gamma Z}, \mathcal{R}_{\gamma \gamma}\right)$ plane which satisfy the vacuum stability requirements in Eq. (9), the constraints from $W$ and $Z$ decays in Eq. (12), and the parameter limitations in Eq. (13). We notice that many of the $\mathcal{R}_{\gamma \gamma}$ values are close to 1 and within the allowed ranges from ATLAS and CMS. The plot also reveals that for the $\mathcal{R}_{\gamma \gamma}$ points compatible with

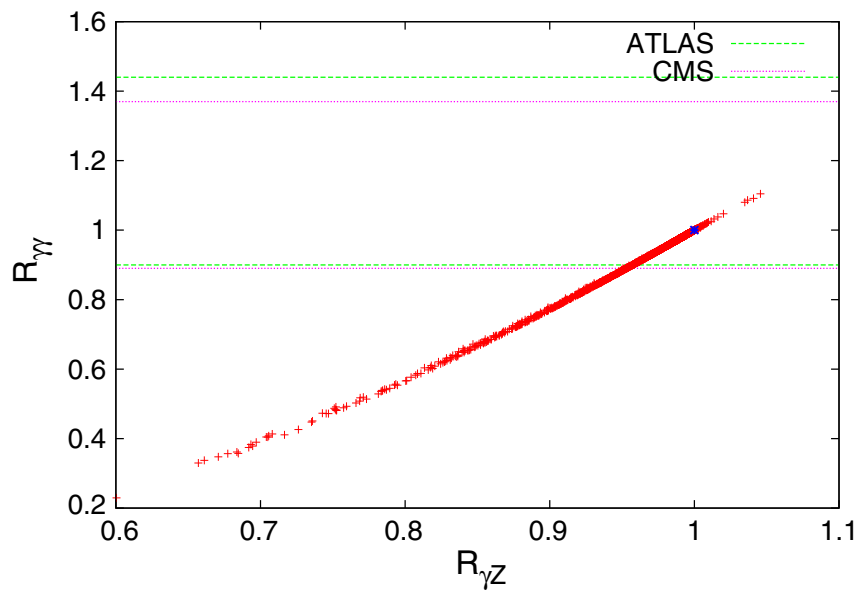

FIG. 2 (color online). The effects of the new charged scalars $H_{1,2}^{ \pm}$on the ratios of the rates of Higgs decay channels $h \rightarrow \gamma \gamma$ and $h \rightarrow \gamma Z$ to their respective SM values for 5000 benchmark points as described in the text. The blue point marks the SM value. The region between the green (magenta) horizontal lines represents the one-sigma range of the ATLAS (CMS) data [24,31].

the LHC data the values of $\Gamma(h \rightarrow \gamma Z)$ do not differ from its SM value by more than $10 \%$ or so. Furthermore, there is a positive correlation between $\mathcal{R}_{\gamma \gamma}$ and $\mathcal{R}_{\gamma Z}$, which is much like the situations in a different recent model with two inert doublets [9] and in the case of only one inert doublet $[33,35,36]$. This can be checked experimentally when the $\gamma Z$ mode is observed in the future.

\section{ELECTROWEAK PRECISION TESTS}

The interactions of the new doublets with the SM gauge bosons described by Eq. (10) bring about modifications, $\Delta S$ and $\Delta T$, to the so-called oblique electroweak parameters $S$ and $T$ which encode the effects of new physics not directly coupled to SM fermions [37]. At the one-loop level $[3,37]$

$$
\begin{aligned}
\frac{\alpha \Delta S}{4 c_{\mathrm{w}}^{2} s_{\mathrm{w}}^{2}} & =\frac{A_{Z Z}\left(m_{Z}^{2}\right)-A_{Z Z}(0)}{m_{Z}^{2}}-A_{\gamma \gamma}^{\prime}(0)-\frac{c_{\mathrm{w}}^{2}-s_{\mathrm{w}}^{2}}{c_{\mathrm{w}} s_{\mathrm{w}}} A_{\gamma Z}^{\prime}(0), \\
\alpha \Delta T & =\frac{A_{W W}(0)}{m_{W}^{2}}-\frac{A_{Z Z}(0)}{m_{Z}^{2}},
\end{aligned}
$$

where the functions $A_{X Y}\left(q^{2}\right)$ can be extracted from the vacuum polarization tensors $\Pi_{X Y}^{\mu \nu}\left(q^{2}\right)=A_{X Y}\left(q^{2}\right) g^{\mu \nu}+$ $\left[q^{\mu} q^{\nu}\right.$ terms] of the SM gauge bosons due to the new scalars' loop contributions, and $A_{X Y}^{\prime}(0)=\left[d A_{X Y}\left(q^{2}\right) /\right.$ $\left.d q^{2}\right]_{q^{2}=0}$. In our numerical analysis below, we will impose

$$
\Delta S=0.05 \pm 0.11, \quad \Delta T=0.09 \pm 0.13,
$$

which are based on the results of a recent fit [38] to electroweak precision data for a Higgs mass $m_{h}=125 \mathrm{GeV}$. 


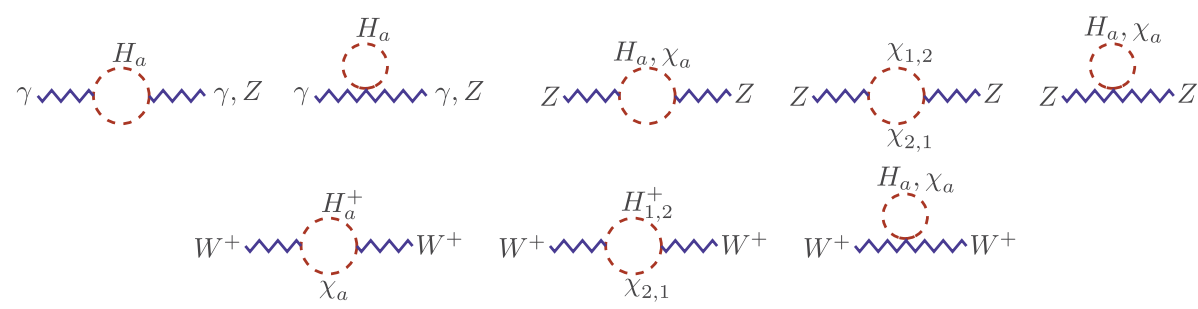

FIG. 3 (color). Feynman diagrams for the contributions of the inert scalar doublets to the oblique electroweak parameters $\Delta S$ and $\Delta T$.
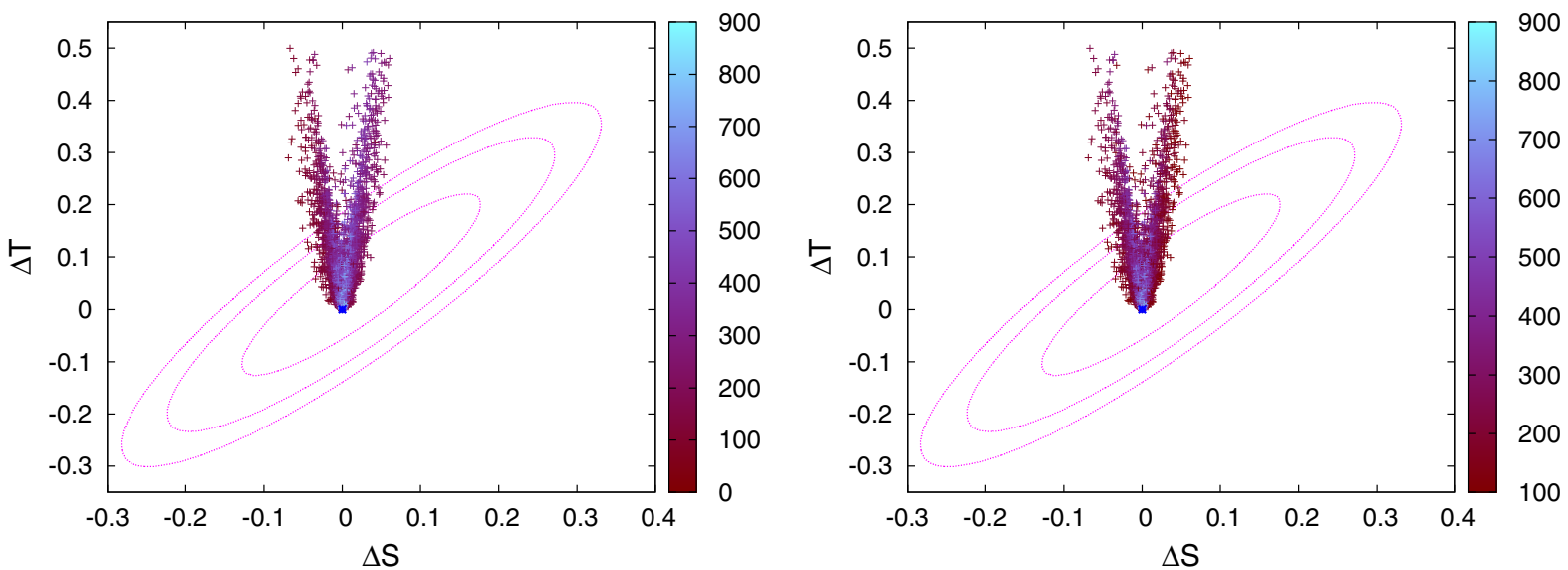

FIG. 4 (color online). The contributions of the inert scalar doublets to the oblique electroweak parameters $\Delta S$ and $\Delta T$ for the 5000 benchmarks used previously. On the left panel, the palette belongs to the lighter neutral inert scalar's mass, $m_{\chi_{1}}$, in GeV. On the right panel, the palette belongs to the lighter charged scalar mass, $m_{H_{1}}$, in $\mathrm{GeV}$. The different contours represent $68 \%$, $95 \%$, and $99 \%$ confidence level, respectively. The blue point at $(0,0)$ marks the SM value.

The contributions of the inert scalars to $\Delta S$ and $\Delta T$ arise from the diagrams depicted in Fig. 3. After evaluating them, we arrive at ${ }^{4}$

$$
\begin{aligned}
\Delta S=\frac{1}{6 \pi}[ & \ln \frac{m_{\chi_{1}} m_{\chi_{2}}}{m_{H_{1}^{ \pm}} m_{H_{2}^{ \pm}}}+s_{2 \theta}^{2} \frac{22 m_{\chi_{1}}^{2} m_{\chi_{2}}^{2}-5 m_{\chi_{1}}^{4}-5 m_{\chi_{2}}^{4}}{6\left(m_{\chi_{1}}^{2}-m_{\chi_{2}}^{2}\right)^{2}} \\
+ & \left.s_{2 \theta}^{2} \frac{\left(m_{\chi_{1}}^{2}+m_{\chi_{2}}^{2}\right)\left(m_{\chi_{1}}^{4}-4 m_{\chi_{1}}^{2} m_{\chi_{2}}^{2}+m_{\chi_{2}}^{4}\right)}{\left(m_{\chi_{1}}^{2}-m_{\chi_{2}}^{2}\right)^{3}} \ln \frac{m_{\chi_{1}}}{m_{\chi_{2}}}\right] \\
\Delta T= & \frac{1}{8 \alpha \pi^{2} v^{2}}\left[c_{\theta}^{2} \mathcal{F}\left(m_{H_{1}^{ \pm}}, m_{\chi_{1}}\right)\right. \\
& +c_{\theta}^{2} \mathcal{F}\left(m_{H_{2}^{ \pm}}, m_{\chi_{2}}\right)+s_{\theta}^{2} \mathcal{F}\left(m_{H_{1}^{ \pm}}, m_{\chi_{2}}\right) \\
& \left.+s_{\theta}^{2} \mathcal{F}\left(m_{H_{2}^{ \pm}}, m_{\chi_{1}}\right)-4 c_{\theta}^{2} s_{\theta}^{2} \mathcal{F}\left(m_{\chi_{1}}, m_{\chi_{2}}\right)\right]
\end{aligned}
$$

where

$$
\mathcal{F}(m, n)=\frac{m^{2}+n^{2}}{2}-\frac{m^{2} n^{2}}{m^{2}-n^{2}} \ln \frac{m^{2}}{n^{2}}
$$

\footnotetext{
${ }^{4}$ Their counterparts in the case of only one inert scalar doublet were computed in Ref. [39].
}

In Fig. 4, we present the distribution on the $(\Delta S, \Delta T)$ plane of the inert scalars' contributions for the 5000 benchmarks employed previously for Fig. 2. Evidently, it is possible for the masses of the charged scalars to be as small as $100 \mathrm{GeV}$ and still be compatible with the electroweak precision measurements. However, we find that the lighter one of the inert neutral scalars, $\chi_{1}$, must be heavier than about $90 \mathrm{GeV}$, which is a stronger condition than that inferred from the LEP constraint on the invisible width of the $Z$ boson. This also makes the bound from the data on the Higgs invisible/nonstandard decay irrelevant.

\section{HIGGS TRILINEAR COUPLING}

Since the new scalars couple directly to the Higgs boson, their presence can cause its trilinear self-coupling, $\lambda_{h h h}$, to shift from its SM prediction. Such a modification could translate into detectable collider signatures, especially at a future $e^{+} e^{-}$machine such as the International Linear Collider [40] where the coupling can be measured with $20 \%$ precision or better at a center-of-mass energy $\sqrt{s}=$ $500 \mathrm{GeV}$ if the integrated luminosity is $500 \mathrm{fb}^{-1}$.

To derive the formula for the mass-dimension Higgs trilinear self-coupling in the presence of extra heavy particles, we follow the steps taken in Ref. [41]. It is just the third derivative of the Higgs effective potential, namely 


$$
\lambda_{h h h}=\left.\frac{\partial^{3}}{\partial \varphi^{3}} V_{\mathrm{eff}}^{T=0}(\varphi)\right|_{\varphi=v}
$$

where $\varphi$ is the classical Higgs field and $V_{\mathrm{eff}}^{T=0}(\varphi)$ is the potential evaluated at temperature $T=0$. We estimate the potential at the one-loop level in the so-called $\overline{\mathrm{DR}}^{\prime}$ scheme $[42,43]$ where it has the form

$V_{\mathrm{eff}}^{T=0}(\varphi)=\frac{\mu_{1}^{2}}{2} \varphi^{2}+\frac{\lambda_{1}}{8} \varphi^{4}+\sum_{i} n_{i} \frac{\left(m_{i}^{2}(\varphi)\right)^{2}}{64 \pi^{2}}\left(\ln \frac{m_{i}^{2}(\varphi)}{\Lambda^{2}}-\frac{3}{2}\right)$.

In the sum above, the index $i$ runs over all the contributing particles, $n_{i}$ stands for the number of internal degrees of freedom of the $i$ th particle, with a minus sign added if it is a fermion, $m_{i}^{2}(\varphi)$ is its field-dependent squared mass, and $\Lambda$ is the renormalization scale which we choose to be the Higgs mass, $\Lambda=125.1 \mathrm{GeV}$. More explicitly, $n_{h}=1, n_{\mathcal{G}}=n_{Z}=n_{\gamma}=3, n_{W}=6, n_{t}=-12$, and $n_{\chi_{a}}=n_{H_{a}^{ \pm}}=2$, where $\mathcal{G}$ refers to the Goldstone bosons. We have collected the formulas for the various relevant $m_{i}^{2}(\varphi)$ in Appendix $\mathrm{C}$.

At tree level we have $\mu_{1}^{2}=-\lambda_{1} v^{2} / 2 \equiv \hat{\mu}_{1}^{2}$, but it receives the one-loop correction

$$
\delta \mu_{1}^{2}=-\left.\frac{1}{32 \pi^{2} v} \sum_{i} n_{i} m_{i}^{2} \dot{m}_{i}^{2}\left(\ln \frac{m_{i}^{2}}{\Lambda^{2}}-1\right)\right|_{\varphi=v},
$$

which follows from $\partial V_{\mathrm{eff}}^{T=0}(\varphi) / \partial \varphi=0$ set at $\varphi=v \simeq$ $246 \mathrm{GeV}$, where $m_{i}^{2} \equiv m_{i}^{2}(\varphi)$ and $\dot{m}_{i}^{2} \equiv \partial m_{i}^{2} / \partial \varphi$. Then the Higgs mass at the one-loop level, which is nothing but the second derivative of $V_{\mathrm{eff}}^{T=0}(\varphi)$, is given by

$$
\begin{aligned}
m_{h}^{2}= & \lambda_{1} v^{2}+\sum_{i} \frac{n_{i}}{32 \pi^{2}}\left[\left(\ddot{m}_{i}^{2} m_{i}^{2}-\frac{\dot{m}_{i}^{2} m_{i}^{2}}{v}+\left(\dot{m}_{i}^{2}\right)^{2}\right)\right. \\
& \left.\times \ln \frac{m_{i}^{2}}{\Lambda^{2}}-\ddot{m}_{i}^{2} m_{i}^{2}+\frac{\dot{m}_{i}^{2} m_{i}^{2}}{v}\right]\left.\right|_{\varphi=v},
\end{aligned}
$$

where the first term is the familiar tree-level contribution, the second term is the radiative one-loop correction, and $\ddot{m}_{i}^{2} \equiv \partial^{2} m_{i}^{2} / \partial \varphi^{2}$. Accordingly, with $m_{h}^{2}$ being fixed to its empirical value, as $\lambda_{1}$ is varied along with the other scalar couplings it can be bigger or smaller than its tree-level value $\hat{\lambda}_{1}=m_{h}^{2} / v^{2} \simeq 0.258$, depending on the size and sign of the loop contribution in $\mathrm{Eq}(30)$.

Incorporating Eq. (30) into Eq. (27), one then obtains

$$
\begin{aligned}
\lambda_{h h h}= & \frac{3 m_{h}^{2}}{v}+\frac{1}{32 \pi^{2}} \sum_{i=\mathrm{all}} n_{i}\left\{\left[\dddot{m}_{i}^{2} m_{i}^{2}+3\left(\dot{m}_{i}^{2}-\frac{m_{i}^{2}}{v}\right)\right.\right. \\
& \left.\times\left(\ddot{m}_{i}^{2}-\frac{\dot{m}_{i}^{2}}{v}\right)\right] \ln \frac{m_{i}^{2}}{\Lambda^{2}}+\frac{\left(\dot{m}_{i}^{2}\right)^{3}}{m_{i}^{2}}-\dddot{m}_{i}^{2} m_{i}^{2} \\
& \left.+\frac{3 m_{i}^{2}}{v}\left(\ddot{m}_{i}^{2}-\frac{\dot{m}_{i}^{2}}{v}\right)\right\}\left.\right|_{\varphi=v},
\end{aligned}
$$

where $\dddot{m}_{i}^{2} \equiv \partial^{3} m_{i}^{2} / \partial \varphi^{3}$. Its SM counterpart, $\lambda_{h h h}^{\mathrm{SM}}$, has the same formula, except that in the sum $i$ runs over SM fields only.

According to Eq. (31) and Appendix C, the Higgs trilinear self-coupling is a function of the couplings $\lambda_{3 a}+\lambda_{4 a}$ and $\lambda_{3 a}$ of the inert neutral and charged scalars, respectively, to the SM Higgs doublet, i.e., through the field-dependent masses and their derivatives. Since $\lambda_{3 a, 4 a}$ are related to the scalars' physical masses via Eqs. (4) and (6), the Higgs trilinear coupling also depends on them. To illustrate how the inert scalars' couplings and masses affect $\lambda_{h h h}$, we define the relative change

$$
\Delta=\frac{\lambda_{h h h}-\lambda_{h h h}^{\mathrm{SM}}}{\lambda_{h h h}^{\mathrm{SM}}}
$$

with respect to the SM prediction. Then in Fig. 5 we graph $\Delta$ versus $\left|\lambda_{32}+\lambda_{42}\right|$ and $\left|\lambda_{32}\right|$, respectively, for the 5000 benchmark points employed earlier. On the same plots we also show the mass distributions of the inert neutral and charged scalars, respectively.

It is clear that in the presence of the inert doublets the trilinear Higgs self-coupling can be enhanced or reduced by up to roughly $150 \%$ relative to the SM contribution to it. One realizes that, for either large or small (charged and/or neutral) scalar masses and couplings to the SM Higgs doublet, this enhancement or reduction of the trilinear coupling is the effect of the superposition of different contributions which could be constructive or destructive.

The new scalars' impact can be further seen in Fig. 6, which illustrates their loop effects. Specifically, it displays the relative changes of the trilinear Higgs coupling, the Higgs mass, and the parameter $\mu_{1}^{2}$ due to radiative corrections versus the Higgs quartic self-coupling $\lambda_{1}$, where

$$
\delta \lambda_{h h h}=\lambda_{h h h}-3 \lambda_{1} v, \quad \delta m_{h}^{2}=m_{h}^{2}-\lambda_{1} v^{2},
$$

$\delta \mu_{1}^{2}$ is defined in Eq. (29), and $\mu_{1}^{2}=\hat{\mu}_{1}^{2}+\delta \mu_{1}^{2}$.

We remark that the Higgs quartic self-coupling, which at tree level is defined by the Higgs mass, can have a wide range from about $10^{-4}$ to 0.5 . This is due to the fact that much of the Higgs mass arises radiatively, as the right plot in Fig. 6 indicates. More precisely, $m_{h}$ can be fully radiative for small $\lambda_{1}$ values or get a negative radiative correction for large $\lambda_{1}$ values, those greater than its tree-level one, $\hat{\lambda}_{1}$. One can see from the top-left and bottom plots in the figure that similar remarks could be made concerning $\lambda_{h h h}$ and $\mu_{1}^{2}$. In particular, each of these parameters may be fully radiative for small $\lambda_{1}$ and also can receive radiative corrections which are negative. 

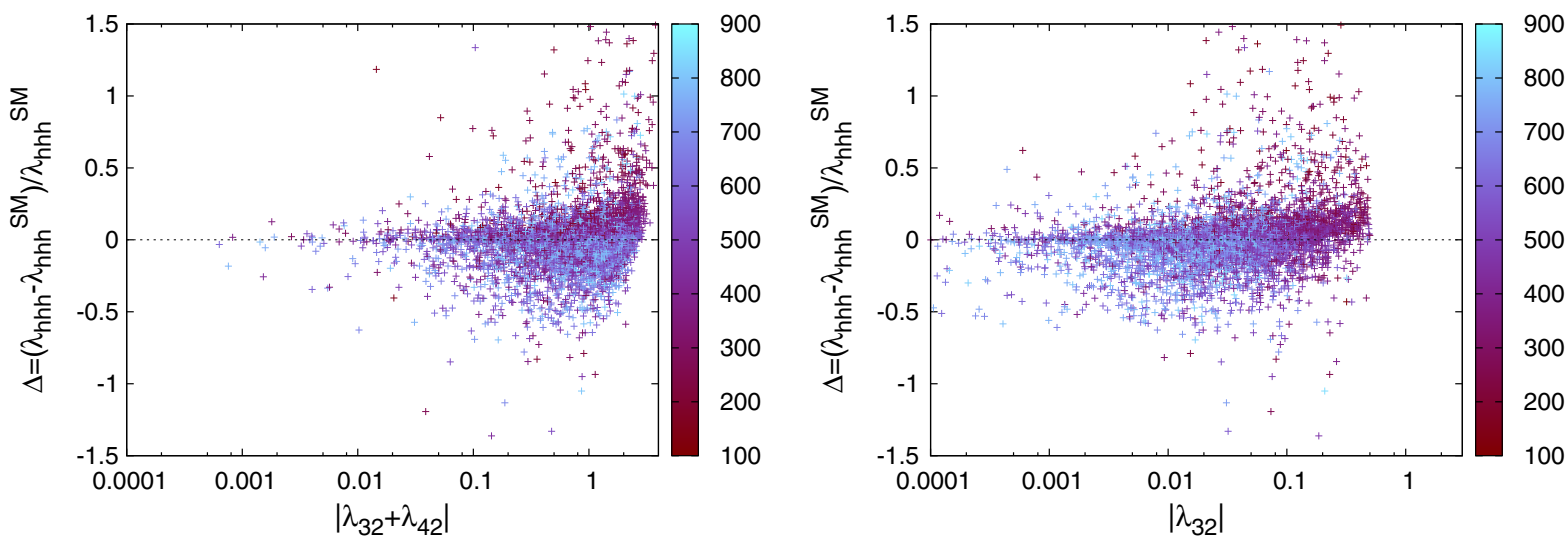

FIG. 5 (color online). The changes of the Higgs trilinear self-coupling relative to its SM value versus the absolute values of the SM Higgs doublet couplings, $\lambda_{32}+\lambda_{42}$ and $\lambda_{32}$, to the heavy neutral (left) and the heavy charged (right) scalars, respectively. On the palettes, we read the heavy neutral (left) and charged (right) scalar masses in $\mathrm{GeV}$.

\section{ELECTROWEAK PHASE TRANSITION}

It is well known that one of the reasons why the SM fails to produce successful baryogenesis [44] is the fact that the EWPT is not strong and consequently cannot suppress processes that violate the conservation of baryon plus lepton numbers, $B+L$, in the broken phase [45]. The suppression of anomalous $B+L$-violating processes in the broken phase happens if the criterion for strongly firstorder EWPT [46,47],

$$
v_{c} / T_{c}>1,
$$
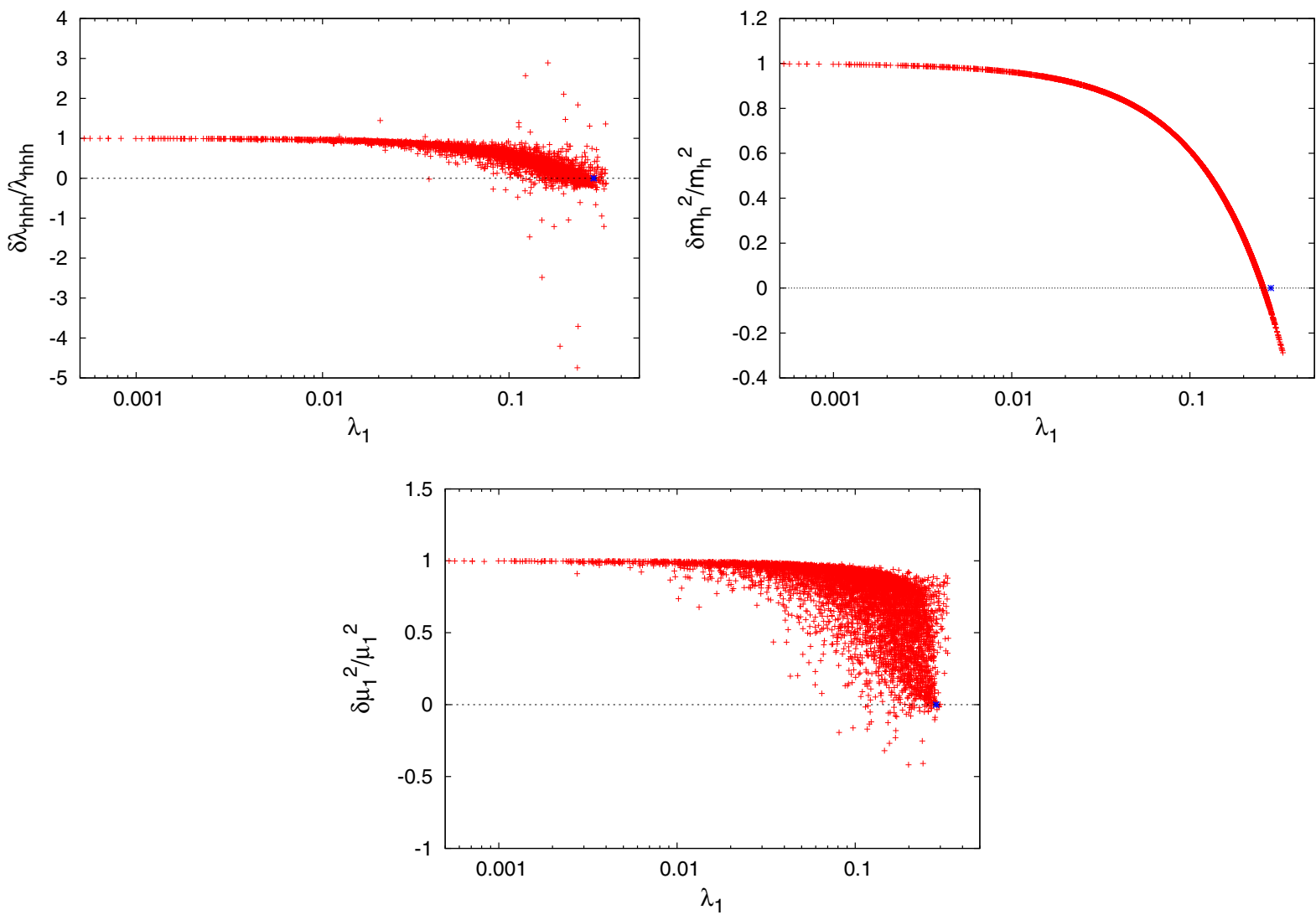

FIG. 6 (color online). The relative changes of the trilinear Higgs self-coupling (left), the Higgs mass (right) and the $\mu_{1}^{2}$ parameter (bottom) due to loop corrections versus the Higgs quartic self-coupling $\lambda_{1}$. The Higgs mass $m_{h}$ is fixed to $125.1 \mathrm{GeV}$. The blue points represent the SM values. 
is fulfilled, where $v_{c}$ is the Higgs VEV at the critical temperature $T_{c}$ at which the effective potential exhibits two degenerate minima, one at zero and the other at $v_{c}$. Both $T_{c}$ and $v_{c}$ are determined using the full thermal effective potential $[48,49]$

$$
V_{\mathrm{eff}}(\varphi, T)=V_{\mathrm{eff}}^{T=0}(\varphi)+\frac{T^{4}}{2 \pi^{2}} \sum_{i} n_{i} J_{\mathrm{B}, \mathrm{F}}\left(m_{i}^{2}(\varphi) / T^{2}\right)
$$

at a finite temperature $T$, where

$$
J_{\mathrm{B}, \mathrm{F}}(r)=\int_{0}^{\infty} d x x^{2} \ln \left[1 \mp \exp \left(-\sqrt{x^{2}+r}\right)\right]
$$

the upper (lower) sign referring to a boson (fermion). To $V_{\text {eff }}(\varphi, T)$ one should add the so-called daisy (or ring) contribution [50]

$$
V_{\text {ring }}(\varphi, T)=-\frac{T}{12 \pi} \sum_{i} n_{i}\left(\tilde{m}_{i}^{3}(\varphi, T)-m_{i}^{3}(\varphi)\right)
$$

which represents the leading term of higher-order loop corrections that may play an important role during the EWPT dynamics. In $V_{\text {ring }}(\varphi, T)$ the sum is over the scalar and longitudinal gauge degrees of freedom, $\tilde{m}_{i}^{2}(\varphi, T)=$ $m_{i}^{2}(\varphi)+\Pi_{i}(T)$ are their thermal squared masses, and $\Pi_{i}(T)$ are the thermal parts of the self energies, which are collected in Appendix C. To estimate $V_{\text {ring }}(\varphi, T)$, one performs the resummation of an infinite class of infrareddivergent multiloops diagrams, known as ring diagrams, that describes the dominant contribution of long distances and gives a significant contribution when (almost) massless states appear in the system. In our case, we will include this by following another approach. Rather than adding $V_{\text {ring }}(\varphi, T)$ to $V_{\text {eff }}(\varphi, T)$, we will replace in Eq. (35) the field-dependent masses of the scalar and longitudinal gauge degrees of freedom with their thermal masses $\tilde{m}_{i}(\varphi, T)$.

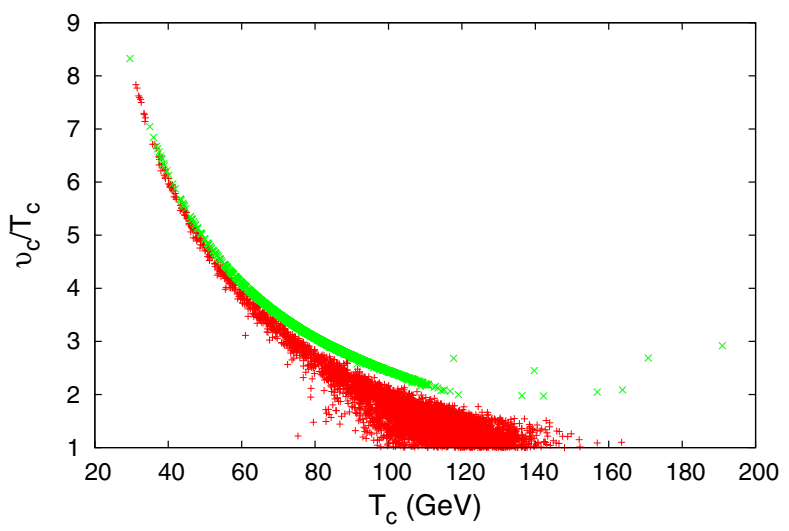

In the criterion for a strong first-order phase transition, Eq. (34), the critical temperature $T_{c}$ is the value at which the two minima of the effective potential are degenerate,

$$
\begin{aligned}
& \left.\frac{\partial}{\partial \varphi} V_{\text {eff }}\left(\varphi, T_{c}\right)\right|_{\varphi=v_{c}}=0, \\
& V_{\text {eff }}\left(\varphi=v_{c}, T_{c}\right)=V_{\text {eff }}\left(\varphi=0, T_{c}\right) .
\end{aligned}
$$

In the SM, this leads to a Higgs mass below $42 \mathrm{GeV}$ [51], since the ratio $v_{c} / T_{c}$ is inversely proportional to the Higgs quartic coupling $\lambda_{1}$. The strength of the EWPT can be improved if new bosonic degrees of freedom are invoked [52-55], which is the case we are investigating. It is clear from Eq. (30) that for large values of the couplings and/or masses of the extra scalars, the one-loop corrections to the Higgs mass could be significant, which allows $\lambda_{1}$ to be smaller and, therefore, fulfills the criterion in Eq. (34) without conflicting with the recent Higgs mass measurements [23,24]. Here, the relevant couplings are those of the Higgs doublet to the charged scalars, $\lambda_{3 a}$, and to the neutral ones, $\lambda_{3 a}+\lambda_{4 a}$, in the limit $\left|\lambda_{5}\right| \ll\left|\lambda_{3 a, 4 a}\right|$. The situation may be compared to those in similar setups [56-59] where extra scalars can help bring about a strongly first-order EWPT by (i) relaxing the Higgs quartic coupling $\lambda_{1}$ to as small as $\mathcal{O}\left(10^{-4}\right)$ and (ii) enhancing the value of the effective potential at the wrong vacuum at the critical temperature without suppressing the ratio $v_{c} / T_{c}$, which relaxes the severe bound on the mass of the SM Higgs.

The integral in Eq. (36) is often approximated by a high temperature expansion. However, in order to take into account the effect of all the (heavy and light) degrees of freedom, we will evaluate them numerically.

With the same 5000 benchmark points used previously, in Fig. 7 we present $v_{c} / T_{c}$ as a function of $T_{c}$ and of the Higgs quartic self-coupling. It is obvious that the criterion for a strongly first-order EWPT is easily satisfied for a large number of benchmarks. Moreover, we find that the daisy contribution to the effective potential tends to weaken the

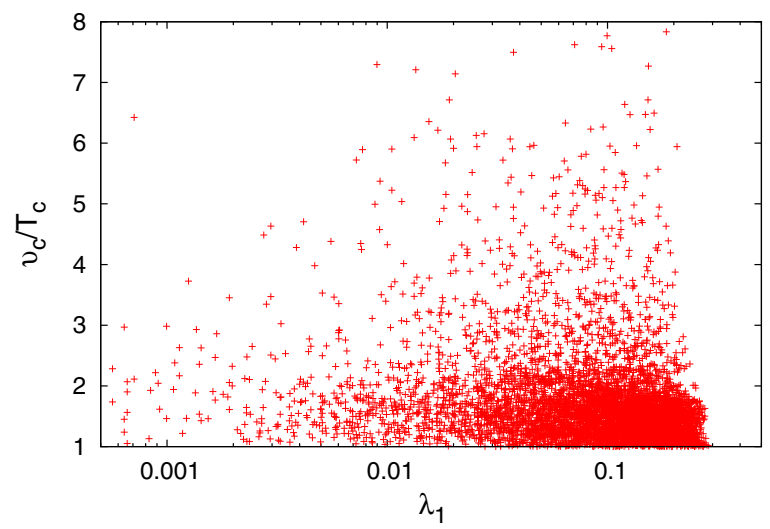

FIG. 7 (color online). Left panel: $v_{c} / T_{c}$ versus $T_{c}$, estimated with (red) and without (green) the daisy contribution. Right panel: $v_{c} / T_{c}$ versus the Higgs quartic self-coupling $\lambda_{1}$, estimated by considering the daisy contribution. 
EWPT strength in this setup. One also notices that a strong EWPT can be obtained for different values of the Higgs quartic self-coupling $\lambda_{1}$, as shown in the right panel of Fig. 7, even for values larger than the tree-level one, $\hat{\lambda}_{1}$. This leads us to conclude that the EWPT is always strongly first-order due the reason (ii) mentioned above, where the extra heavy scalars' existence makes the Higgs VEV slowly varying with respect to temperature and the wrong vacuum value, i.e., $V_{\text {eff }}(\varphi=0, T)$, is evolving and increases with temperature.

We remark that due to the absence of a $C P$-violating phase in the potential $\mathcal{V}$ an additional source of $C P$ violation has to be included in the Lagrangian of the more complete theory for it to be realistic for baryogensis. One possibility is to introduce dimension-six operators which couple the inert scalars to the top-quark mass and are suppressed by a new-physics scale that can be well above one $\mathrm{TeV}$, in analogy to a scenario of electroweak baryogenesis from a singlet scalar [60].
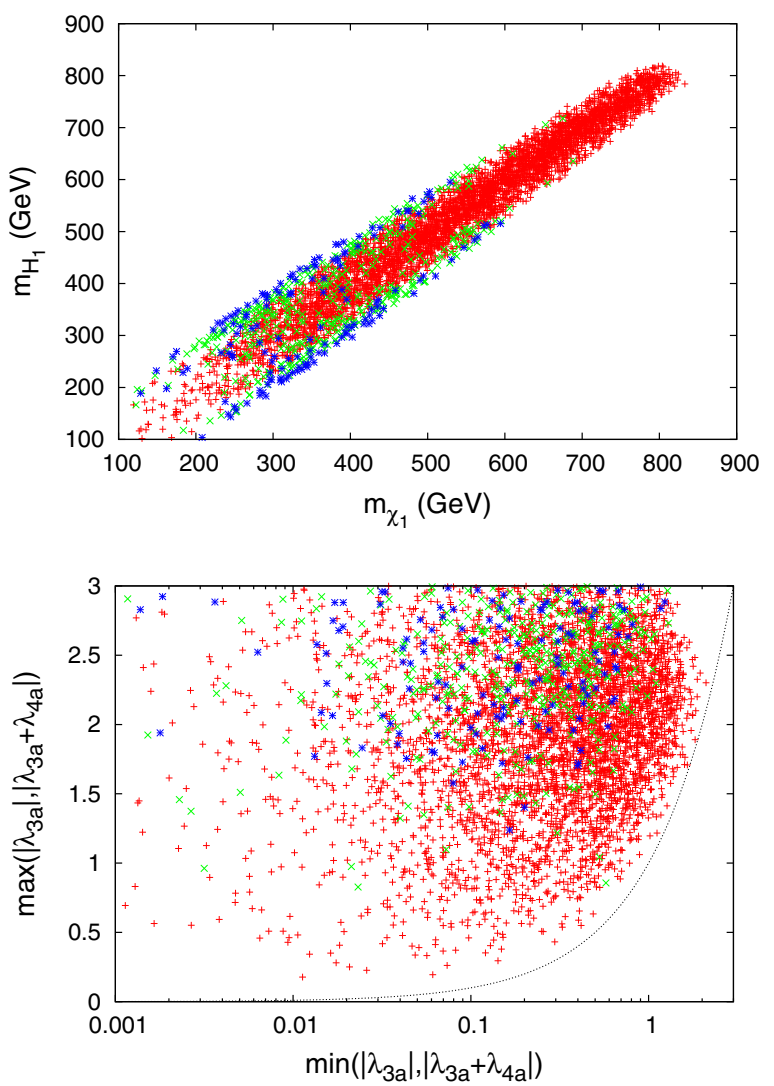

\section{DISCUSSION AND CONCLUSION}

According to the analysis carried out in previous sections, the extra scalars can have important effects on the Higgs phenomenology and the electroweak phase transition if these particles are relatively light and the couplings to the SM Higgs doublet are large $\left(\lambda_{3 a}\right.$ for charged scalars and $\lambda_{3 a}+\lambda_{4 a}$ for neutral ones). Therefore, from the 5000 benchmark points used previously, we extract those that simultaneously satisfy (i) the constraint from the measurements on the Higgs decay mode $h \rightarrow \gamma \gamma$, namely $0.9<\mathcal{R}_{\gamma \gamma}<1.37$, (ii) the electroweak precision tests, i.e., all the points inside the three ellipsoids in Fig. 4, and (iii) the criterion $v_{c} / T_{c}>1$ for strongly first-order EWPT. As mentioned in Sec. IV, the Higgs decay channel into a pair of inert scalars is closed for all the viable benchmarks and hence its experimental bound is not relevant. Here, we divide the points fulfilling the conditions (i,ii,iii) into three sets according to the ellipsoid to which they belong on the $(\Delta S, \Delta T)$ plane. The results are displayed in Fig. 8 .
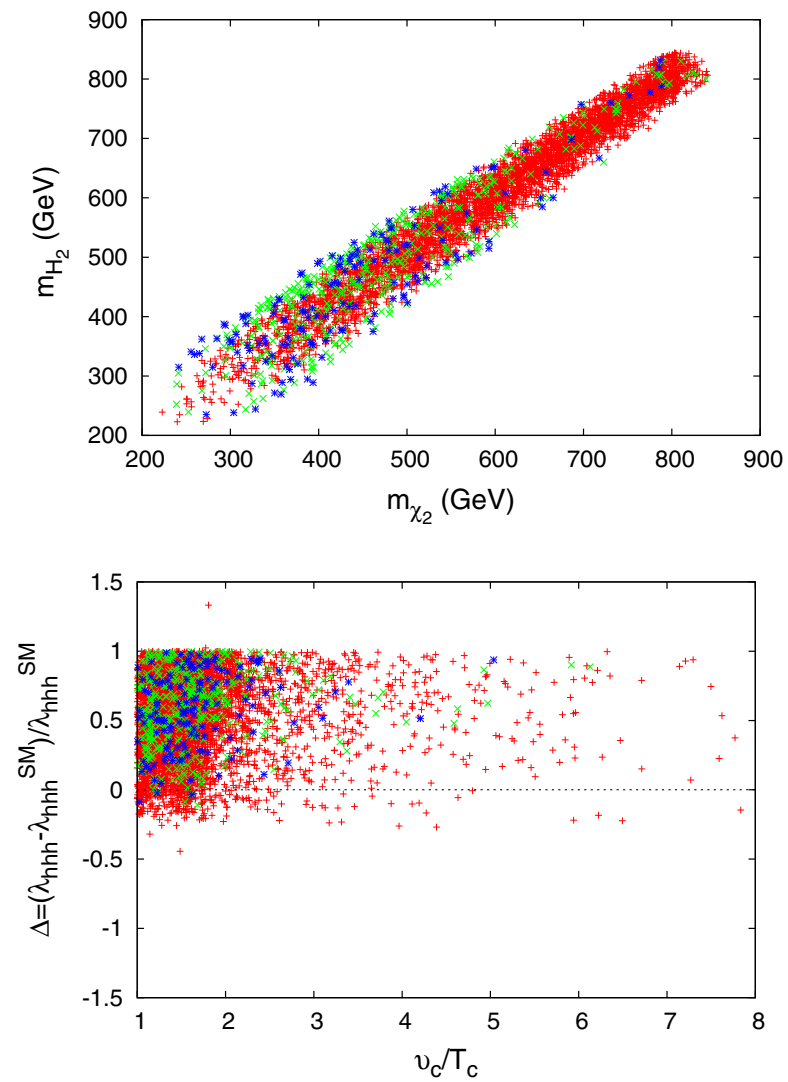

FIG. 8 (color online). Top panels: the distribution of masses of the extra charged and neutral scalars under the assumptions described in the text. Bottom left panel: the strength distribution of the different quartic self-coupling of the Higgs doublet to the extra charged and neutral scalars; the dashed line represents the case where the couplings are equal in magnitude, i.e., $\operatorname{Min}\left(\left|\lambda_{3 a}\right|,\left|\lambda_{3 a}+\lambda_{4 a}\right|\right)=\operatorname{Max}\left(\left|\lambda_{3 a}\right|,\left|\lambda_{3 a}+\lambda_{4 a}\right|\right)$. Bottom right panel: the EWPT strength versus the relative enhancement of the Higgs trilinear self-coupling. The red, green, and blue points correspond to those inside the 99\%, 95\%, and 68\% CL ellipsoids on the $(\Delta S, \Delta T)$ plane in Fig. 4. 
From the top panels in Fig. 8, one can see that the extra scalar masses do not exceed $900 \mathrm{GeV}$ according to our parameter choices in Eq. (13). The charged scalars could be light up to the LEP II bound (100 GeV), while the neutral scalars, which were supposed to be less constrained before, are now not allowed to be less than $120 \mathrm{GeV}$ due to the electroweak precision tests in this model. From the bottom left panel, it is evident that the couplings of the Higgs doublet to the charged scalars, $\lambda_{3 a}$, and to the neutral ones, $\lambda_{3 a}+\lambda_{4 a}$, could be both larger than 1 or smaller than 0.5 . They could vary also within the whole considered range [0:3], or they could be almost equal in absolute values (i.e., close to the dashed curve). The bottom right plot in this figure reveals that, while strongly first-order EWPT occurs for all of the viable benchmark points, only for some of them there is a positive correlation between the EWPT strength and substantial enhancement of the Higgs trilinear self-coupling relative to the SM prediction as shown in Ref. [15].

In conclusion, we have considered a scenario beyond the SM involving three scalar weak doublets and investigated a number of implications of the case where two of the doublets are inert and charged under a dark Abelian gauge symmetry. We looked at the effects of the new scalars on oblique electroweak parameters, the Higgs decay modes $h \rightarrow \gamma \gamma, \gamma Z$, and its trilinear coupling. We also examined how the inert scalars can induce strongly first-order EWPT. Taking into account various theoretical and experimental constraints, we demonstrated that the viable parameter space can all accommodate strongly first-order EWPT and contains regions in which the Higgs trilinear selfcoupling is enhanced/reduced by up to $150 \%$ compared to its SM value. Future experiments with sufficient precision can test the new scalars' effects that we have obtained on the Higgs decays $h \rightarrow \gamma \gamma, \gamma Z$ and trilinear coupling.

\section{ACKNOWLEDGMENTS}

We would like to thank Masaya Kohda for helpful discussions. A. A. is supported by the Algerian Ministry of Higher Education and Scientific Research under the CNEPRU Project No. D01720130042. The work of G. F. and J. T. was supported in part by the research Grant No. NTU-ERP-102R7701, the MOE Academic Excellence
Program (Grant No. 102R891505), and the National Center for Theoretical Sciences of Taiwan.

\section{APPENDIX A: VACUUM STABILITY CONDITIONS}

We can rewrite the doublets $\Phi$ and $\eta_{1,2}$ and their products according to

$$
\begin{aligned}
\Phi & =f \hat{\Phi}, \quad \hat{\Phi}^{\dagger} \hat{\Phi}=1, \quad \eta_{a}=e_{a} \hat{\eta}_{a}, \\
\hat{\eta}_{a}^{\dagger} \hat{\eta}_{a} & =1, \quad f, e_{a}>0, \\
\hat{\Phi}^{\dagger} \hat{\eta}_{a} \hat{\eta}_{a}^{\dagger} \hat{\Phi} & =\rho_{a}, \quad \hat{\eta}_{1}^{\dagger} \hat{\eta}_{2} \hat{\eta}_{2}^{\dagger} \hat{\eta}_{1}=\rho^{\prime}, \quad 0 \leq \rho_{a}, \rho^{\prime} \leq 1 .
\end{aligned}
$$

Assuming that $\lambda_{5}$ in Eq. (2) is negligible compared to the other $\lambda$ 's, we can then express the part of $\mathcal{V}$ that is quartic in the doublets approximately as

$$
\begin{aligned}
\mathcal{V}_{4}= & \frac{1}{2} \lambda_{1} f^{4}+\frac{1}{2} \lambda_{21} e_{1}^{4}+\frac{1}{2} \lambda_{22} e_{2}^{4}+\lambda_{31} f^{2} e_{1}^{2}+\lambda_{32} f^{2} e_{2}^{2} \\
& +\lambda_{41} f^{2} e_{1}^{2} \rho_{1}+\lambda_{42} f^{2} e_{2}^{2} \rho_{2}+\lambda_{6} e_{1}^{2} e_{2}^{2}+\lambda_{7} e_{1}^{2} e_{2}^{2} \rho^{\prime} \\
= & \frac{1}{2}\left(\begin{array}{lll}
f^{2} & e_{1}^{2} & e_{2}^{2}
\end{array}\right) \tilde{\lambda}\left(\begin{array}{c}
f^{2} \\
e_{1}^{2} \\
e_{2}^{2}
\end{array}\right),
\end{aligned}
$$

where

$\tilde{\lambda}=\left(\begin{array}{ccc}\lambda_{1} & \lambda_{31}+\rho_{1} \lambda_{41} & \lambda_{32}+\rho_{2} \lambda_{42} \\ \lambda_{31}+\rho_{1} \lambda_{41} & \lambda_{21} & \lambda_{6}+\rho^{\prime} \lambda_{7} \\ \lambda_{32}+\rho_{2} \lambda_{42} & \lambda_{6}+\rho^{\prime} \lambda_{7} & \lambda_{22}\end{array}\right)$.

To ensure the stability of the vacuum, we need to derive relations among the $\lambda$ 's in $\mathcal{V}_{4}$, which dominates $\mathcal{V}$ at large fields, such that the minimum of $\mathcal{V}_{4}$ remains positive. This can be achieved using copositivity criteria [61], which in this case are applied to the minimum of $\tilde{\lambda}$. Since $\lambda_{4 a, 7}$ can be positive, zero, or negative and $0 \leq \rho_{a}, \rho^{\prime} \leq 1$, we have

$$
\tilde{\lambda}_{\min }=\left(\begin{array}{ccc}
\lambda_{1} & \lambda_{31}+\operatorname{Min}\left(0, \lambda_{41}\right) & \lambda_{32}+\operatorname{Min}\left(0, \lambda_{42}\right) \\
\lambda_{31}+\operatorname{Min}\left(0, \lambda_{41}\right) & \lambda_{21} & \lambda_{6}+\operatorname{Min}\left(0, \lambda_{7}\right) \\
\lambda_{32}+\operatorname{Min}\left(0, \lambda_{42}\right) & \lambda_{6}+\operatorname{Min}\left(0, \lambda_{7}\right) & \lambda_{22}
\end{array}\right) .
$$

From the criteria for strictly copositive $3 \times 3$ matrices [62-64] then follow the conditions in Eq. (9).

\section{APPENDIX B: INTERACTION TERMS FOR $\mathcal{S}_{a}$ AND $\mathcal{P}_{a}$}

The interaction terms of $\chi_{1,2}$ in Eqs. (10) and (14) can be rewritten in terms of the real and imaginary components defined in Eq. (7). Thus 


$$
\begin{aligned}
\mathcal{L} \supset & \frac{g}{2 c_{\mathrm{w}}}\left[c_{2 \theta}\left(\mathcal{P}_{1} \stackrel{\leftrightarrow}{\partial}^{\mu} \mathcal{S}_{1}-\mathcal{P}_{2} \stackrel{\leftrightarrow}{\partial}^{\mu} \mathcal{S}_{2}\right)+s_{2 \theta}\left(\mathcal{P}_{1} \stackrel{\leftrightarrow}{\partial}^{\mu} \mathcal{S}_{2}+\mathcal{P}_{2} \stackrel{\leftrightarrow}{\partial}^{\mu} \mathcal{S}_{1}\right)\right] Z_{\mu}+\frac{i g}{2}\left\{\left[c_{\theta} H_{1}^{+} \stackrel{\leftrightarrow}{\partial}^{\mu}\left(\mathcal{S}_{1}-i \mathcal{P}_{1}\right)+c_{\theta} H_{2}^{+} \stackrel{\leftrightarrow}{\partial}^{\mu}\left(\mathcal{S}_{2}+i \mathcal{P}_{2}\right)\right.\right. \\
& \left.\left.+s_{\theta} H_{1}^{+} \stackrel{\leftrightarrow}{\partial}^{\mu}\left(\mathcal{S}_{2}-i \mathcal{P}_{2}\right)-s_{\theta} H_{2}^{+} \stackrel{\leftrightarrow}{\partial}^{\mu}\left(\mathcal{S}_{1}+i \mathcal{P}_{1}\right)\right] W_{\mu}^{-}-\text {H.c. }\right\}+\frac{g^{2}}{4}\left(\mathcal{S}_{1}^{2}+\mathcal{P}_{1}^{2}+\mathcal{S}_{2}^{2}+\mathcal{P}_{2}^{2}\right)\left(\frac{Z^{2}}{2 c_{\mathrm{w}}^{2}}+W^{+\mu} W_{\mu}^{-}\right) \\
& +\frac{h}{v}\left[\left(c_{\theta}^{2} \mu_{21}^{2}+s_{\theta}^{2} \mu_{22}^{2}-m_{\chi_{1}}^{2}\right)\left(\mathcal{S}_{1}^{2}+\mathcal{P}_{1}^{2}\right)+\left(c_{\theta}^{2} \mu_{22}^{2}+s_{\theta}^{2} \mu_{21}^{2}-m_{\chi_{2}}^{2}\right)\left(\mathcal{S}_{2}^{2}+\mathcal{P}_{2}^{2}\right)+s_{2 \theta}\left(\mu_{21}^{2}-\mu_{22}^{2}\right)\left(\mathcal{S}_{1} \mathcal{S}_{2}+\mathcal{P}_{1} \mathcal{P}_{2}\right)\right] .
\end{aligned}
$$

\section{APPENDIX C: FIELD-DEPENDENT AND THERMAL MASSES}

To estimate the Higgs effective potential, one needs the field-dependent squared masses $m_{i}^{2}(\varphi)$ of all the contributing particles. One also requires the first, second, and third derivatives of $m_{i}^{2}(\varphi)$ to determine the counterterm $\delta \mu_{1}^{2}$ in Eq. (29), the one-loop correction to the Higgs mass, and the enhancement of the Higgs trilinear selfcoupling.

The field-dependent masses of the electroweak gauge bosons and top quark have their SM values. For the other particles, we have the thermal masses $\tilde{m}_{i} \equiv \tilde{m}_{i}(\varphi, T)$ which are given by

$$
\begin{aligned}
\tilde{m}_{\mathcal{G}}^{2} & =\mu_{1}^{2}+\frac{1}{2} \lambda_{1} \varphi^{2}+\Pi_{\Phi}, \quad \tilde{m}_{h}^{2}=\mu_{1}^{2}+\frac{3}{2} \lambda_{1} \varphi^{2}+\Pi_{\Phi}, \\
\tilde{m}_{H_{a}^{ \pm}}^{2} & =\mu_{2 a}^{2}+\frac{1}{2} \lambda_{3 a} \varphi^{2}+\Pi_{\eta_{a}}, \quad \tilde{m}_{\chi_{1,2}}^{2}=\frac{1}{2}\left(C_{1}+C_{2} \mp \sqrt{R}\right), \\
C_{a} & =\mu_{2 a}^{2}+\frac{1}{2}\left(\lambda_{3 a}+\lambda_{4 a}\right) \varphi^{2}+\Pi_{\eta_{a}}, \\
R & =\left(C_{1}-C_{2}\right)^{2}+4 c^{2}, \quad c=\frac{1}{4}\left|\lambda_{5}\right| \varphi^{2},
\end{aligned}
$$

and related to $m_{i}(\varphi)$ by $\tilde{m}_{i}^{2}(\varphi, T)=m_{i}^{2}(\varphi)+\Pi_{i}$, where $\Pi_{i} \equiv \Pi_{i}(T)$ denote the thermal parts of the self energies and $\Pi_{\Phi, \eta_{a}}$ are listed below. Hence the Goldstone bosons (G) and the Higgs boson also have the same field-dependent masses as their respective counterparts in the SM. We note that the inert $C P$-even and $C P$-odd neutral scalars mix, leading to equal-mass eigenstates, according to Eq. (8).
It is simple to get the first, second, and third derivatives of $m_{i}^{2}(\varphi)$ from Eq. (C1). For completeness, here we supply them explicitly:

$$
\begin{aligned}
\dot{m}_{\mathcal{G}}^{2}(\varphi) & =\lambda_{1} \varphi, \quad \dot{m}_{h}^{2}(\varphi)=3 \lambda_{1} \varphi, \quad \dot{m}_{H_{a}^{ \pm}}^{2}(\varphi)=\lambda_{3 a} \varphi, \\
\dot{m}_{\chi_{1,2}}^{2}(\varphi) & =\frac{1}{2}\left(\dot{C}_{1}+\dot{C}_{2} \mp \frac{\dot{R}}{2 \sqrt{R}}\right), \\
\dot{C}_{a} & =\left(\lambda_{3 a}+\lambda_{4 a}\right) \varphi, \quad \dot{c}=\frac{1}{2}\left|\lambda_{5}\right| \varphi, \\
\dot{R} & =2\left(\dot{C}_{1}-\dot{C}_{2}\right)\left(C_{1}-C_{2}\right)+8 \dot{c} c, \\
\ddot{m}_{\mathcal{G}}^{2}(\varphi)= & \lambda_{1}, \quad \ddot{m}_{h}^{2}(\varphi)=3 \lambda_{1}, \quad \ddot{m}_{H_{a}^{ \pm}}^{2}(\varphi)=\lambda_{3 a}, \\
\ddot{m}_{\chi_{1,2}}^{2}(\varphi)= & \frac{1}{2}\left(\ddot{C}_{1}+\ddot{C}_{2} \mp \frac{\ddot{R}}{2 \sqrt{R}} \pm \frac{\dot{R}^{2}}{4 \sqrt{R^{3}}}\right), \\
\ddot{C}_{a}= & \lambda_{3 a}+\lambda_{4 a}, \quad \ddot{c}=\frac{1}{2}\left|\lambda_{5}\right|, \\
\ddot{R}= & 2\left(\dot{C}_{1}-\dot{C}_{2}\right)^{2}+2\left(\ddot{C}_{1}-\ddot{C}_{2}\right)\left(C_{1}-C_{2}\right) \\
& +8 \dot{c}^{2}+8 \ddot{c}_{c}, \quad(\mathrm{C} 3) \\
\dddot{m}_{\mathcal{G}}^{2}(\varphi) & =\dddot{m}_{h}^{2}(\varphi)=\dddot{m}_{H_{a}^{ \pm}}^{2}(\varphi)=\dddot{C}_{a}=\dddot{c}=0, \\
\dddot{m}_{\chi_{1,2}}^{2}(\varphi)= & \mp \frac{1}{4 \sqrt{R}}\left(\dddot{R}^{2}-\frac{3 \dot{R} \ddot{R}}{2 R}+\frac{3 \dot{R}^{3}}{4 R^{2}}\right), \\
& \dddot{R}=6\left(\ddot{C}_{1}-\ddot{C}_{2}\right)\left(\dot{C}_{1}-\dot{C}_{2}\right)+24 \ddot{c} \dot{c} .
\end{aligned}
$$

Finally, we write down the thermal parts $\Pi_{i}$ of the pertinent self-energies. For the scalar and electroweak bosons [65]

$$
\begin{aligned}
& \Pi_{\Phi}=\left(6 \lambda_{1}+\frac{9}{4} g^{2}+\frac{3}{4} g_{Y}^{2}+3 y_{t}^{2}+4 \lambda_{31}+2 \lambda_{41}+4 \lambda_{32}+2 \lambda_{42}\right) \frac{T^{2}}{12}, \\
& \Pi_{\eta_{a}}=\left(\frac{9}{4} g^{2}+\frac{3}{4} g_{Y}^{2}+4 \lambda_{3 a}+2 \lambda_{4 a}+6 \lambda_{2 a}+4 \lambda_{6}+2 \lambda_{7}+\frac{3}{4} \mathcal{Q}_{\eta_{a}}^{2} g_{D}^{2}\right) \frac{T^{2}}{12}, \\
& \Pi_{W}=\frac{17}{6} g^{2} T^{2}, \quad \Pi_{B}=\frac{11}{16} g_{Y}^{2} T^{2},
\end{aligned}
$$

where $y_{t}$ denotes the top-quark Yukawa coupling and $\mathcal{Q}_{\eta_{a}}=\mathcal{Q}_{C} \eta_{a}$ is the charge of the inert doublet $\eta_{a}$ under $\mathrm{U}(1)_{D}$. Numerically, since $g_{D}$ is unknown, for definiteness we set $g_{D}=g_{Y}$. 
[1] G. Aad et al. (ATLAS Collaboration), Observation of a new particle in the search for the standard model Higgs boson with the ATLAS detector at the LHC, Phys. Lett. B 716, 1 (2012).

[2] S. Chatrchyan et al. (CMS Collaboration), Observation of a new boson at a mass of $125 \mathrm{GeV}$ with the CMS experiment at the LHC, Phys. Lett. B 716, 30 (2012).

[3] K. A. Olive et al. (Particle Data Group Collaboration), Review of particle physics (RPP), Chin. Phys. C 38, 090001 (2014).

[4] J. F. Gunion, H. E. Haber, G. L. Kane, and S. Dawson, The Higgs Hunter's Guide (Westview Press, Colorado, 2000).

[5] G. C. Branco, P. M. Ferreira, L. Lavoura, M. N. Rebelo, M. Sher, and J. P. Silva, Theory and phenomenology of twoHiggs-doublet models, Phys. Rep. 516, 1 (2012).

[6] I. P. Ivanov, V. Keus, and E. Vdovin, Abelian symmetries in multi-Higgs-doublet models, J. Phys. A 45, 215201 (2012).

[7] A. C. B. Machado and V. Pleitez, Natural flavour conservation in a three Higg-doublet model, arXiv:1205.0995.

[8] V. Keus, S. F. King, and S. Moretti, Three-Higgs-doublet models: Symmetries, potentials and Higgs boson masses, J. High Energy Phys. 01 (2014) 052.

[9] E. C. F. S. Fortes, A. C. B. Machado, and J. Montaño, and V. Pleitez, Correlated prediction of $h \rightarrow \gamma Z$ from $h \rightarrow \gamma \gamma$ at LHC, arXiv:1408.0780.

[10] M. Maniatis and O. Nachtmann, Stability and symmetry breaking in the general three-Higgs-doublet model, J. High Energy Phys. 02 (2015) 058.

[11] I. P. Ivanov and C. C. Nishi, Symmetry breaking patterns in 3HDM, J. High Energy Phys. 01 (2015) 021.

[12] S. Moretti and K. Yagyu, Constraints on parameter space from perturbative unitarity in models with three scalar doublets, Phys. Rev. D 91, 055022 (2015).

[13] V. Keus, S. F. King, S. Moretti, and D. Sokolowska, Dark matter with two inert doublets plus one Higgs doublet, J. High Energy Phys. 11 (2014) 016.

[14] E. Ma, I. Picek, and B. Radovcić, New scotogenic model of neutrino mass with $U(1)_{D}$ gauge interaction, Phys. Lett. B 726, 744 (2013).

[15] S. Kanemura, Y. Okada, and E. Senaha, Electroweak baryogenesis and quantum corrections to the triple Higgs boson coupling, Phys. Lett. B 606, 361 (2005).

[16] A. Noble and M. Perelstein, Higgs self-coupling as a probe of electroweak phase transition, Phys. Rev. D 78, 063518 (2008).

[17] C. W. Chiang and T. Yamada, Electroweak phase transition in Georgi-Machacek model, Phys. Lett. B 735, 295 (2014).

[18] K. Fuyuto and E. Senaha, Improved sphaleron decoupling condition and the Higgs coupling constants in the real singlet-extended standard model, Phys. Rev. D 90, 015015 (2014).

[19] D. Curtin, P. Meade, and C. T. Yu, Testing electroweak baryogenesis with future colliders, J. High Energy Phys. 11 (2014) 127.

[20] S. Kanemura, T. Kasai, and Y. Okada, Mass bounds of the lightest $C P$ even Higgs boson in the two Higgs doublet model, Phys. Lett. B 471, 182 (1999).
[21] S. Y. Ho and J. Tandean, Probing scotogenic effects in $e+$ $e$ - colliders, Phys. Rev. D 89, 114025 (2014).

[22] E. Ma, Verifiable radiative seesaw mechanism of neutrino mass and dark matter, Phys. Rev. D 73, 077301 (2006).

[23] G. Aad et al. (ATLAS Collaboration), Measurement of the Higgs boson mass from the $H \rightarrow \gamma \gamma$ and $H \rightarrow Z Z^{*} \rightarrow 4 \ell$ channels with the ATLAS detector using $25 \mathrm{fb}^{-1}$ of $p p$ collision data, Phys. Rev. D 90, 052004 (2014).

[24] CMS Collaboration, Report No. CMS-PAS-HIG-14-009, July 2014.

[25] D. Curtin, R. Essig, S. Gori, P. Jaiswal, A. Katz, T. Liu, Z. Liu, D. McKeen et al., Exotic decays of the $125 \mathrm{GeV}$ Higgs boson, Phys. Rev. D 90, 075004 (2014).

[26] A. Falkowski, F. Riva, and A. Urbano, Higgs at last, J. High Energy Phys. 11 (2013) 111.

[27] P. P. Giardino, K. Kannike, I. Masina, M. Raidal, and A. Strumia, The universal Higgs fit, J. High Energy Phys. 05 (2014) 046.

[28] J. Ellis and T. You, Updated global analysis of Higgs couplings, J. High Energy Phys. 06 (2013) 103.

[29] G. Belanger, B. Dumont, U. Ellwanger, J. F. Gunion, and S. Kraml, Global fit to Higgs signal strengths and couplings and implications for extended Higgs sectors, Phys. Rev. D 88, 075008 (2013).

[30] K. Cheung, J. S. Lee, and P. Y. Tseng, Higgs precision analysis updates 2014, Phys. Rev. D 90, 095009 (2014).

[31] G. Aad et al. (ATLAS Collaboration), Measurement of Higgs boson production in the diphoton decay channel in pp collisions at center-of-mass energies of 7 and $8 \mathrm{TeV}$ with the ATLAS detector, Phys. Rev. D 90, 112015 (2014).

[32] https://twiki.cern.ch/twiki/bin/view/LHCPhysics/ CERNYellowReportPageBR3.

[33] S. Y. Ho and J. Tandean, Probing scotogenic effects in Higgs boson decays, Phys. Rev. D 87, 095015 (2013).

[34] C. S. Chen, C. Q. Geng, D. Huang, and L. H. Tsai, New scalar contributions to $h \rightarrow Z \gamma$, Phys. Rev. D 87, 075019 (2013).

[35] B. Swiezewska and M. Krawczyk, Diphoton rate in the inert doublet model with a $125 \mathrm{GeV}$ Higgs boson, Phys. Rev. D 88, 035019 (2013).

[36] A. D. Banik and D. Majumdar, Inert doublet dark matter with an additional scalar singlet and $125 \mathrm{GeV}$ Higgs boson, Eur. Phys. J. C 74, 3142 (2014).

[37] M.E. Peskin and T. Takeuchi, Estimation of oblique electroweak corrections, Phys. Rev. D 46, 381 (1992).

[38] M. Baak, J. Cúth, J. Haller, A. Hoecker, R. Kogler, K. Mönig, M. Schott, and J. Stelzer, The global electroweak fit at NNLO and prospects for the LHC and ILC, Eur. Phys. J. C 74, 3046 (2014).

[39] R. Barbieri, L. J. Hall, and V.S. Rychkov, Improved naturalness with a heavy Higgs: An alternative road to LHC physics, Phys. Rev. D 74, 015007 (2006).

[40] H. Baer et al., Physics at the International Linear Collider, available at: http://lcsim.org/papers/DBDPhysics.pdf.

[41] A. Ahriche, A. Arhrib, and S. Nasri, Higgs phenomenology in the two-singlet model, J. High Energy Phys. 02 (2014) 042 .

[42] A. B. Lahanas and D. V. Nanopoulos, The road to no scale supergravity, Phys. Rep. 145, 1 (1987). 
[43] S. P. Martin, Two loop effective potential for a general renormalizable theory and softly broken supersymmetry, Phys. Rev. D 65, 116003 (2002).

[44] V. A. Kuzmin, V. A. Rubakov, and M. E. Shaposhnikov, On the anomalous electroweak baryon number nonconservation in the early universe, Phys. Lett. 155B, 36 (1985).

[45] M. Trodden, Electroweak baryogenesis, Rev. Mod. Phys. 71, 1463 (1999).

[46] M. E. Shaposhnikov, Baryon asymmetry of the universe in standard electroweak theory, Nucl. Phys. B287, 757 (1987).

[47] M.E. Shaposhnikov, Structure of the high temperature gauge ground state and electroweak production of the baryon asymmetry, Nucl. Phys. B299, 797 (1988).

[48] L. Dolan and R. Jackiw, Symmetry behavior at finite temperature, Phys. Rev. D 9, 3320 (1974).

[49] S. Weinberg, Gauge and global symmetries at high temperature, Phys. Rev. D 9, 3357 (1974).

[50] M. E. Carrington, The effective potential at finite temperature in the standard model, Phys. Rev. D 45, 2933 (1992).

[51] A. I. Bochkarev and M. E. Shaposhnikov, Electroweak production of baryon asymmetry and upper bounds on the Higgs and top masses, Mod. Phys. Lett. A 02, 417 (1987).

[52] A. Ahriche and S. Nasri, Electroweak phase transition in the U(1)'-MSSM, Phys. Rev. D 83, 045032 (2011).

[53] T. A. Chowdhury, M. Nemevsek, G. Senjanovic, and Y. Zhang, Dark matter as the trigger of strong electroweak phase transition, J. Cosmol. Astropart. Phys. 02 (2012) 029.
[54] D. Borah and J. M. Cline, Inert doublet dark matter with strong electroweak phase transition, Phys. Rev. D 86, 055001 (2012).

[55] G. Gil, P. Chankowski, and M. Krawczyk, Inert dark matter and strong electroweak phase transition, Phys. Lett. B 717, 396 (2012).

[56] A. Ahriche and S. Nasri, Dark matter and strong electroweak phase transition in a radiative neutrino mass model, J. Cosmol. Astropart. Phys. 07 (2013) 035.

[57] A. Ahriche, What is the criterion for a strong first order electroweak phase transition in singlet models?, Phys. Rev. D 75, 083522 (2007).

[58] A. Ahriche and S. Nasri, Light dark matter, light Higgs and the electroweak phase transition, Phys. Rev. D 85, 093007 (2012).

[59] A. Ahriche, K. L. McDonald, and S. Nasri (to be published).

[60] J. M. Cline and K. Kainulainen, Electroweak baryogenesis and dark matter from a singlet Higgs, J. Cosmol. Astropart. Phys. 01 (2013) 012.

[61] K. Kannike, Vacuum stability conditions from copositivity criteria, Eur. Phys. J. C 72, 2093 (2012).

[62] K. Hadeler, On copositive matrices, Linear Algebra Appl. 49, 79 (1983).

[63] G. Chang and T. W. Sederberg, Nonnegative quadratic Bézier triangular patches, Comput. Aided Geom. Des. 11, 113 (1994).

[64] L. Ping and F. Y. Yu, Criteria for copositive matrices of order four, Linear Algebra Appl. 194, 109 (1993).

[65] J. I. Kapusta and C. Gale, Finite-Temperature Field Theory: Principles and Applications (Cambridge University Press, Cambridge, England, 2006). 